\title{
Top-quark polarization and asymmetries at the LHC in the effective description of squark interactions
}

\author{
A. Abrahantes ${ }^{a}$, E. Arganda ${ }^{b}$, S. Peñaranda ${ }^{c}$ \\ Departamento de Física Teórica, Facultad de Ciencias, Universidad de Zaragoza, 50009 Zaragoza, Spain
}

Received: 10 June 2014 / Accepted: 11 January 2015 / Published online: 29 January 2015

(C) The Author(s) 2015. This article is published with open access at Springerlink.com

\begin{abstract}
A detailed study of top-quark polarizations and $t \bar{t}$ charge asymmetries, induced by top-squark-pair production at the LHC and the subsequent decays $\tilde{t} \rightarrow t \tilde{\chi}_{1}^{0}$, is performed within the effective description of squark interactions, which includes the effective Yukawa couplings and another logarithmic term encoding the supersymmetry breaking. This effective approach is more suitable for its introduction into Monte-Carlo simulations and we make use of its implementation in MadGraph in order to investigate the possibilities of the charge asymmetry $A_{\mathrm{C}}$, measured at the LHC and consistent with SM expectations, to discriminate between different SUSY scenarios and analyze the implications of these scenarios in the top polarizations and related observables.
\end{abstract}

\section{Introduction}

The Standard Model (SM) of the electroweak and strong interactions is the present paradigm of particle physics and provides a very good description of all data collected so far at hadron and lepton colliders [1], including the recent discovery of the SM-like Higgs boson at the LHC [2,3]. However, there are arguments against the SM being the fundamental model of particle interactions [4], giving rise to the investigation of competing extended models. Among the alternatives of physics beyond the SM, one of the most promising possibilities is supersymmetry (SUSY) [5-8], which leads to a renormalizable field theory with precisely calculable predictions to be tested in present and future experiments and whose simplest realization at the electroweak scale is the Minimal Supersymmetric Standard Model (MSSM) [9]. The MSSM predicts the existence of superpartners for each SM particle: squarks/sleptons, gauginos and higgsinos are the partners of quarks/leptons, gauge and higgs bosons, respectively.

\footnotetext{
a e-mail: arian@unizar.es

be-mail: ernesto.arganda@unizar.es

c e-mail: siannah@unizar.es
}

The Higgs sector contains two scalars doublets, with a spectrum that includes three neutral Higgs bosons $(h, H, A)$ and one charged Higgs pair $\left(H^{ \pm}\right)[10]$, with the lightest MSSM Higgs boson $h$ being completely consistent with the discovered SM-like Higgs boson with mass $m_{h_{\mathrm{SM}}}=125.9 \mathrm{GeV}$ (see e.g. [11]).

In this work we focus on the properties of the top squarks, the SUSY partners of SM top quarks. In particular, we concentrate on the top-squark decay channels involving neutralinos, the fermionic neutral superpartners of the electroweak gauge and Higgs bosons. Once produced, top squarks will decay in a way dependent on the model parameters (see e.g. [12]). If the decay channels into gluinos and quarks or into other squarks and gauge or Higgs bosons are not kinematically allowed, the main decay channels of top squarks are their partial decays into charginos and $b$ quarks $\left(\tilde{t} \rightarrow b \tilde{\chi}^{ \pm}\right)$ or into neutralinos and top quarks $\left(\tilde{t} \rightarrow t \tilde{\chi}^{0}\right)$. Some of those channels are expected to be always open, given the large mass difference between quarks and top squarks, and that the charginos/neutralinos are expected to be lighter than the top squarks in the majority of SUSY-breaking models. In the few cases in which these channels are closed, the top squarks will decay through flavor-changing neutral channels [13-15], or through three- or four-body decay channels involving a non-resonant SUSY particle [16-21]. Here we will concentrate on the top-squark decay channels involving neutralinos and top quarks, within the effective description developed in [22], more suitable for their introduction in the Monte-Carlo programs used in experimental analysis. This computation combines the effective description (which includes higher order terms) with the complete oneloop description (which includes all kinetic and mass-effects factors) and defines a new effective coupling. It includes a non-decoupling logarithmic gluino mass term, which implies a deviation of the higgsino/gaugino and Higgs/gauge couplings equality predicted by exact SUSY. This deviation is important and has to be taken into account in the experimen- 
tal measurement of SUSY relations. It is showed in [22] that the effective description approximates the improved description within a $10 \%$ precision, except in special uninteresting corners of the parameter space, where the corresponding branching ratios are practically zero. Whilst the results in [22] apply the description only to squark decays, a more recent work [23] expands the results of [22] by applying those results to the production cross section of squarks at the LHC. In [23,24], this effective description has been implemented in MadGraph [25,26] MSSM framework [27], in order to be applied to the partial decay widths of squarks into charginos and neutralinos and compute the corresponding cross sections.

After the discovery of the top-quark [28,29], top-quark physics has entered the era of precision measurements. Among the many measurements performed, the $t \bar{t}$ forwardbackward asymmetry has received a special attention because of a disagreement with respect to the SM predictions [30-37]. The $t \bar{t}$ lepton-based asymmetries are above the SM as well [38-41]. These discrepancies have motivated a plethora of new physics explanations. However, most of the present precision $t \bar{t}$ measurements at the LHC exclude some of the simplest forms of the new physics models proposed. In this work we analyze some effects of SUSY in new physics $t \bar{t}$ observables.

An important aspect of the top-squark phenomenology is the possible contribution of the decay channel $\tilde{t} \rightarrow t \tilde{\chi}_{1}^{0}$ to new physics $t \bar{t}$ observables at the LHC [42-45], as charge asymmetries or top-quark polarization. The presence of topquarks from this decay could mean non-zero polarizations in the resulting final state at the LHC. Since the top-quark decay occurs before hadronization, the polarization can have important implications for the kinematic distributions of the final particles, and hence on the search strategies for the top squarks [45]. The longitudinal polarization of top-quarks coming from top-squark decays into neutralinos depends on the mass difference between the top-squark and the neutralino, as well as on the mixing in both sectors, and can take any value between -1 and +1 , while the measurements of ATLAS [46] and CMS [47] are in good agreement with the SM prediction of negligible top-quark polarization. Therefore, polarization studies may supply information as regards different SUSY scenarios [48]. On the other hand, explanations of the top quark forward-backward asymmetry $A_{\mathrm{FB}}$ observed at the Tevatron $[38,39,49-51]$, which exceeds the SM predictions [30-34,36,37,52], must take into account the measurements of the $t \bar{t}$ charge asymmetry $A_{\mathrm{C}}$ at the LHC [53-55] which are consistent with the SM expectations and tightly correlated with $A_{\mathrm{FB}}$ [56-64], given that both $t \bar{t}$ observables could receive contributions from top-squark pair production decaying into neutralinos and top-quarks. However, it is possible to have an excess in $A_{\mathrm{FB}}$ and no excess in $A_{\mathrm{C}}$ if some cancelation occurs $[60,65-68]$ or even exhibit differences in $t \bar{t} \gamma$ production $[69,70]$. We will not try in this work to search for particular SUSY scenarios which would fit better the asymmetry $A_{\mathrm{FB}}$ measured at the Tevatron and analyze their $A_{\mathrm{C}}$ predictions at the LHC. Our purpose here will be rather to look into the consistency of the proposed SUSY scenarios with the SM expectations for the charge asymmetries defined in [37] and then study the implications of these scenarios on the top-quark polarizations and related observables at the LHC, which may help to discriminate between them and the SM. We put special emphasis on the way the effective description of squarks interactions affects these observables.

The paper is organized as follows. In Sect. 2 we review the most relevant features of the effective description of squark/chargino/neutralino interactions. The new physics $t \bar{t}$ observables related to the top-quark polarizations and charge asymmetries at the LHC are presented in Sect. 3. Section 4 is devoted to the numerical analysis of $t \bar{t}$ charge asymmetries and top-quark polarization observables for several SUSY scenarios, comparing these results to the SM predictions calculated at next to leading order (NLO). In the end, the conclusions and final comments are summarized in Sect. 5.

\section{Theoretical framework: effective description approximation}

Here we work within the MSSM framework. It is well known that QCD corrections to the squark partial decay widths into charginos and neutralinos can be numerically large, specially in certain regions of the parameter space [71-74]. The complete one-loop corrections to squark partial decay widths are already available [74,75], but their complicated expressions are not suitable for the introduction in Monte-Carlo programs used for experimental analysis. An effective description of squark/chargino/neutralino couplings, simple to write and to introduce in computer codes, was given in [22]. This description contains the large one-loop corrections from the finite threshold corrections to the quark masses, but it also contains higher order corrections including another logarithmic term which encodes the supersymmetry breaking. The above effective description have been recently implemented in MadGraph MSSM framework [23]. In this article we use this implementation to discuss the effects of the radiative corrections included in the effective description of squark interactions on top polarization and top-quark asymmetries.

In the following we present the crucial expressions of the effective approach which have been included in MadGraph package $[25,26]$. We briefly introduce the tree-level Lagrangian of interactions of the quark-squarkchargino/neutralino and then, an extract of the analysis in [22] depicting the effective description of the squarks interactions is presented. The tree-level interaction Lagrangian between fermion-sfermion-(chargino or neutralino) reads [74] 


$$
\begin{aligned}
\mathcal{L}_{\chi \tilde{f} f^{\prime}} & =\sum_{a=1,2} \sum_{r} \mathcal{L}_{\chi_{r} \tilde{f}_{a} f^{\prime}}+\text { h.c. }, \\
\mathcal{L}_{\chi_{r} \tilde{f}_{a} f^{\prime}} & =-g \tilde{f}_{a}^{*} \bar{\chi}_{r}\left(A_{+a r}^{(f)} P_{L}+A_{-a r}^{(f)} P_{R}\right) f^{\prime} .
\end{aligned}
$$

Here we have adopted a compact notation, where $f^{\prime}$ is either $f$ or its $S U(2)_{L}$ partner for $\chi_{r}$ being a neutralino or a chargino, respectively. Roman characters $a, b \ldots$ are reserved for sfermion indices and $i, j, \ldots$ for chargino indices, Greek indices $\alpha, \beta, \ldots$ denote neutralinos, Roman indices $r, s \ldots$ indicate either a chargino or a neutralino. For example, the top-squark interactions with charginos are obtained by replacing $f \rightarrow t, f^{\prime} \rightarrow b, \chi_{r} \rightarrow \chi_{r}^{-}, r=1,2$. The coupling matrices that encode the dynamics are given by

$$
\begin{aligned}
& A_{+a i}^{(t)}=R_{a 1}^{(t)} V_{i 1}^{*}-\lambda_{t} R_{a 2}^{(t)} V_{i 2}^{*}, \\
& A_{-a i}^{(t)}=-\lambda_{b} R_{a 1}^{(t)} U_{i 2}, \\
& A_{+a \alpha}^{(t)}=\frac{1}{\sqrt{2}}\left(R_{a 1}^{(t)}\left(N_{\alpha 2}^{*}+Y_{L} t_{W} N_{\alpha 1}^{*}\right)+\sqrt{2} \lambda_{t} R_{a 2}^{(t)} N_{\alpha 4}^{*}\right), \\
& A_{-a \alpha}^{(t)}=\frac{1}{\sqrt{2}}\left(\sqrt{2} \lambda_{t} R_{a 1}^{(t)} N_{\alpha 4}-Y_{R}^{t} t_{W} R_{a 2}^{(t)} N_{\alpha 1}\right), \\
& A_{+a i}^{(b)}=R_{a 1}^{(b)} U_{i 1}^{*}-\lambda_{b} R_{a 2}^{(b)} U_{i 2}^{*}, \\
& A_{-a i}^{(b)}=-\lambda_{t} R_{a 1}^{(b)} V_{i 2}, \\
& A_{+a \alpha}^{(b)}=-\frac{1}{\sqrt{2}}\left(R_{a 1}^{(b)}\left(N_{\alpha 2}^{*}-Y_{L} t_{W} N_{\alpha 1}^{*}\right)-\sqrt{2} \lambda_{b} R_{a 2}^{(b)} N_{\alpha 3}^{*}\right), \\
& A_{-a \alpha}^{(b)}=-\frac{1}{\sqrt{2}}\left(-\sqrt{2} \lambda_{b} R_{a 1}^{(b)} N_{\alpha 3}+Y_{R}^{b} t_{W} R_{a 2}^{(b)} N_{\alpha 1}\right)
\end{aligned}
$$

with $Y_{L}$ and $Y_{R}^{t, b}$ the weak hypercharges of the left-handed $S U(2)_{L}$ doublet and right-handed singlet fermion, and $\lambda_{t}$ and $\lambda_{b}$ are the Yukawa couplings.

In the effective description approach, following hints from Higgs-boson physics [76-80], an effective Yukawa coupling is defined as

$$
\begin{aligned}
\lambda_{b}^{\mathrm{eff}} & \equiv \frac{m_{b}^{\mathrm{eff}}}{v_{1}} \equiv \frac{m_{b}(Q)}{v_{1}\left(1+\Delta m_{b}\right)}, \\
\lambda_{t}^{\mathrm{eff}} & \equiv \frac{m_{t}^{\mathrm{eff}}}{v_{2}} \equiv \frac{m_{t}(Q)}{v_{2}\left(1+\Delta m_{t}\right)},
\end{aligned}
$$

with $m_{q}(Q)(q \equiv b, t)$ being the running quark mass and $\Delta m_{q}$ is the finite threshold correction. The SUSY-QCD contributions to $\Delta m_{q}$ are

$$
\begin{aligned}
\Delta m_{b}^{\mathrm{SQCD}} & =\frac{2 \alpha_{s}}{3 \pi} m_{\tilde{g}} \mu \tan \beta I\left(m_{\tilde{b}_{1}}, m_{\tilde{b}_{2}}, m_{\tilde{g}}\right), \\
\Delta m_{t}^{\mathrm{SQCD}} & =\frac{2 \alpha_{s}}{3 \pi} m_{\tilde{g}} \frac{\mu}{\tan \beta} I\left(m_{\tilde{t}_{1}}, m_{\tilde{t}_{2}}, m_{\tilde{g}}\right),
\end{aligned}
$$

where $I(a, b, c)$ is the scalar three-point function at zero momentum transfer,

$$
\begin{aligned}
& I(a, b, c) \\
& =\frac{a^{2} b^{2} \ln \left(a^{2} / b^{2}\right)+b^{2} c^{2} \ln \left(b^{2} / c^{2}\right)+a^{2} c^{2} \ln \left(c^{2} / a^{2}\right)}{\left(a^{2}-b^{2}\right)\left(b^{2}-c^{2}\right)\left(a^{2}-c^{2}\right)} .
\end{aligned}
$$

The effective description of the squark interaction consists in replacing the tree-level quark masses in the couplings defined in Eqs. (2) by the effective Yukawa couplings of Eq. (3), and use this Lagrangian to compute the partial decay width (see [22] for details). A Yukawa-improved decay width computation has been defined in [22] and it showed that the effective description using just the Yukawa threshold corrections of Eq. (3) is not enough for the squark partial decay widths description. The one-loop corrections develop a term which grows as the gluino mass $m_{\tilde{g}}$ [81], which is absent in the effective Yukawa couplings in Eq. (3). Therefore, the QCD corrections to squark partial decay widths produce explicit non-decoupling terms of the sort $\log m_{\tilde{g}}$. To understand those terms a renormalization group analysis is in order [22]. It is possible to construct an effective theory below the gluino mass scale, which contains only squarks, quarks, charginos, neutralinos and gluons in the light sector of the theory, and integrate out the gluino contributions. We calculate the renormalization group equations (RGE) of the gaugino and higgsino couplings, and perform the matching with the full MSSM couplings at the gluino mass scale $m_{\tilde{g}}$. Only the logarithmic RGE effects have been considered, neglecting the possible threshold effects at the gluino mass scale. Since the effective theory does not contain gluinos, only the contributions from the gluon have to be taken into account. In [22], they showed that the effective description (6) approximates the full one-loop computation to within 2-5\% for large enough gluino masses $\left(m_{\tilde{g}} \gtrsim 1 \mathrm{TeV}\right)$. The effects of the new logarithmic terms are more visible in the gauginolike channels, where the Yukawa couplings play no role, and the bulk of the corrections corresponds to the log terms. In the higgsino-like channels their importance is less apparent.

Finally, a simple expression for the effective description of squark/chargino/neutralino couplings is given by [22,23]:

$$
\begin{aligned}
g^{\mathrm{eff}}(Q) & =g\left(\frac{\alpha_{s}(Q)}{\alpha_{s}\left(m_{\tilde{g}}\right)}\right)^{\frac{2}{\beta_{0}}} \simeq g\left(1-\frac{\alpha_{s}(Q)}{\pi} \log \frac{Q}{m_{\tilde{g}}}\right) \\
\tilde{\lambda}_{b, t}^{\mathrm{eff}}(Q) & =\lambda_{b, t}^{\mathrm{eff}}(Q)\left(\frac{\alpha_{s}(Q)}{\alpha_{s}\left(m_{\tilde{g}}\right)}\right)^{\frac{-2}{\beta_{0}}} \\
& \simeq \lambda_{b, t}^{\mathrm{eff}}(Q)\left(1+\frac{\alpha_{s}(Q)}{\pi} \log \frac{Q}{m_{\tilde{g}}}\right),
\end{aligned}
$$

where $\beta_{0}$ is the QCD $\beta$-function and $\lambda^{\text {eff }}(Q)$ are the effective Yukawa couplings, Eq. (3). Then, the effective description of squark interactions consist of replacing the tree-level quark masses and/or gaugino and higgsino couplings in Eq. (2) by the effective couplings as giving above, Eq. (6). 
After introducing these expressions in computer codes as MadGraph, a good description for squark decays into charginos and neutralinos is accomplished, and then we are able to compute any physical processes involving these vertices, including the leading radiative corrections.

\section{New physics $t \bar{t}$ observables}

It is well known that by studying the final states with top quarks at the LHC and measuring the top-quark polarization we are able to differentiate the allowed MSSM scenarios. One possible scenario to explore is the case of the production of two top squarks decaying into neutralinos and tops, $p p \rightarrow$ $\tilde{t}_{i} \tilde{t}_{i}^{*} \rightarrow t \bar{t} \chi \chi$, where $\chi$ stands for $\tilde{\chi}_{1}^{0}, \tilde{\chi}_{2}^{0}$ and $\tilde{t}_{i}$ for $\tilde{t}_{1}, \tilde{t}_{2}$.

The resulting polarization of the top quark coming from the top-squark decays, $\mathcal{P}_{t}$, reads [48]:

$\mathcal{P}_{t}=\frac{\left[\left(A_{-\alpha i}^{(t)}\right)^{2}-\left(A_{+\alpha i}^{(t)}\right)^{2}\right] f_{1}}{\left(A_{-\alpha i}^{(t)}\right)^{2}+\left(A_{+\alpha i}^{(t)}\right)^{2}-2 A_{-\alpha i}^{(t)} A_{+\alpha i}^{(t)} f_{2}}$,

where $f_{1,2}$ are pure kinematic factors given by

$f_{1}=m_{t} \frac{p_{\chi} \cdot s_{t}}{p_{t} \cdot p_{\chi}}, \quad f_{2}=m_{t} \frac{m_{\chi}}{p_{t} \cdot p_{\chi}}$,

with $m_{t}, p_{t}$, and $s_{t}$ denoting the top mass, momentum and longitudinal spin vector, respectively, and $p_{\chi}$ and $m_{\chi}$ the neutralino momentum and mass, and $A_{ \pm \alpha i}^{(t)}$ are the neutralino couplings defined in Eq.(2). In the rest frame of the decaying particle these factor reduce to

$f_{1}=\frac{\lambda^{\frac{1}{2}}\left(m_{\tilde{t}}^{2}, m_{t}^{2}, m_{\chi}^{2}\right)}{m_{\tilde{t}}^{2}-m_{t}^{2}-m_{\chi}^{2}}, \quad f_{2}=\frac{2 m_{t} m_{\chi}}{m_{\tilde{t}}^{2}-m_{t}^{2}-m_{\chi}^{2}}$,

and $\lambda(x, y, z)=x^{2}+y^{2}+z^{2}-2 x y-2 y z-2 x z$. By means of these expressions the polarization of the top quarks can be calculated at tree level and also in the effective approximation of squark interactions.

The measured particle assessing top-quark polarization is the electron coming from the semi-leptonic decay of the $W$ boson from $t \rightarrow b W \rightarrow b l v_{l}$ [42,82-84]. The top-quark polarization enters the lepton angular distribution in the following way [83]:

$\frac{1}{\Gamma_{l}} \frac{d \Gamma_{l}}{d \cos \theta_{l}}=\frac{1}{2}\left(1-\beta_{t}^{2}\right)\left(1-\mathcal{P}_{t} \beta_{t}\right) \frac{1+\frac{\mathcal{P}_{t}-\beta_{t}}{1-\mathcal{P}_{t} \beta_{t}} \cos \theta_{l}}{\left(1-\beta_{t} \cos \theta_{l}\right)^{3}}$,

where $\theta_{l}$ is the angle between the top-quark and the lepton directions in the laboratory frame, and $\beta_{t}$ is the top-quark velocity:
$\beta_{t}=\frac{\left|p_{t}\right|}{E_{t}}$

being $E_{t}$ the total energy of the top quark. The effects from original top-quark polarization, $\mathcal{P}_{t}$, are entangled with the boost in the form of an effective polarization [83]:

$\mathcal{P}_{t}^{\text {eff }}=\frac{\mathcal{P}_{t}-\beta_{t}}{1-\mathcal{P}_{t} \beta_{t}}$.

To measure the top-quark polarization one can define an asymmetry in $\theta_{l}$. Because the $\theta_{l}$ distribution is nonsymmetric, one has the freedom to define asymmetries with respect to different angles, for example [45]

$A_{\theta_{l}}=\frac{\sigma\left(\theta_{l}<\pi / 4\right)-\sigma\left(\theta_{l}>\pi / 4\right)}{\sigma\left(\theta_{l}<\pi / 4\right)+\sigma\left(\theta_{l}>\pi / 4\right)}$,

where $\sigma$ is the integrated cross section.

Another option to characterize the asymmetry is using the azimuthal angle. We define the following axes system: the $\hat{z}$-axis is defined by the proton direction, and the $\hat{x}-\hat{z}$ plane is defined by the top-quark direction and the $\hat{z}$-axis, then $\phi_{l}$ is the azimuthal angle of the lepton in this system. Because at the LHC the initial state has identical particles, the $\hat{z}$-axis can point in the direction of either proton, and it is not possible to distinguish between $\phi_{l}$ and $2 \pi-\phi_{l}$ [48]. We can relate $\theta_{l}$ to $\phi_{l}$ by using the spherical angles coordinates in this axis system: the top-quark angular variables are $\left(\hat{\theta}_{t}, \hat{\phi}_{t}=0\right)$ and the lepton ones are $\left(\hat{\theta}_{l}, \hat{\phi}_{l}=\phi_{l}\right)$, then

$\cos \theta_{l}=\cos \hat{\theta}_{t} \cos \hat{\theta}_{l}+\sin \hat{\theta}_{t} \sin \hat{\theta}_{l} \cos \phi_{l}$,

in this way the lepton distribution (10), after integrating over $\hat{\theta}_{t}$ and $\hat{\theta}_{l}$, picks up a $\phi_{l}$ dependence. We define then the asymmetry [45]

$A_{\phi_{l}}=\frac{\sigma\left(\cos \phi_{l}>0\right)-\sigma\left(\cos \phi_{l}<0\right)}{\sigma\left(\cos \phi_{l}>0\right)+\sigma\left(\cos \phi_{l}<0\right)}$,

where $\sigma$ is the integrated cross section.

Besides, $\beta_{t}, A_{\phi_{l}}$, and $A_{\theta_{l}}$, there are other top-quark-wise observables characterizing the events, such as

$$
\begin{aligned}
& z=\frac{E_{b}}{E_{t}}, \\
& u=\frac{E_{l}}{E_{l}+E_{b}} .
\end{aligned}
$$

The ratios $z$ and $u$, with $E_{t}, E_{b}$, and $E_{l}$ being the labframe energies of the top-quark, and the bottom-quark and the lepton coming from its decay, respectively, are sensitive to the top-quark polarization when the top-quarks are highly boosted [85]. The distributions of these variables can be explored using the MadGraph implementation containing the effective squark approximation [23], allowing us to 
acknowledge whether top-quark polarization observables are sensitive to the effective approximation of squark interactions. It worth mentioning that for our new physics scenario the top-quark polarization is further affected by the underlying event kinematics: our top-quark came from an already boosted system, the squark. Thus, the resulting top-quark polarization has a dependence on the squark boost [45].

Finally, it is well known that the top-quark production at the Tevatron is dominated by the $q \bar{q}$ annihilation, hence the charge asymmetry will be reflected not only in the partonic rest frame but also in the center of mass system of proton and antiproton. The situation is more complex for proton-proton collisions at the LHC, where no preferred direction is at hand in the laboratory frame, thus lacking a natural definition for the charge asymmetry given the symmetric nature of the incoming protons. However, the parton distributions inside the protons are not symmetric for quarks (mainly valence quarks) and antiquarks (all sea quarks), meaning quarks usually carry more momentum than antiquarks. For a positive (negative) charge asymmetry in $q \bar{q} \rightarrow t \bar{t}$ events, the topquark (top-antiquark) is more likely to be produced in the direction of the incoming quark in the $t \bar{t}$ rest frame, resulting in a broader (narrower) rapidity distribution of top-quarks than of top-antiquarks in the laboratory frame. The difference in the absolute values of the rapidities $(y)$ of the top-quarks and antiquarks, $\Delta\left|y_{t}\right|=\left|y_{t}\right|-\left|y_{\bar{t}}\right|$, is therefore a suitable observable to measure the $t \bar{t}$ charge asymmetry. Several processes beyond the SM can alter this asymmetry $[35,56,86-$ 93], either with vector or axial vector couplings or via interference with the SM. Hence the measurement of the charge asymmetry provides a useful tool to test for the presence of new physics that would be hidden in the $t \bar{t}$ invariant mass $\left(m_{t \bar{t}}\right)$ spectrum.

The charge asymmetry in $t \bar{t}$ production at the LHC can be defined as follows:

$A_{C}^{t \bar{t}\left(l^{+} l^{-}\right)}(\xi)=\frac{N(\Delta|\xi|>0)-N(\Delta|\xi|<0)}{N(\Delta|\xi|>0)+N(\Delta|\xi|<0)}$

where $\Delta|\xi|=\left|\xi_{t\left(l^{+}\right)}\right|-\left|\xi_{\bar{t}\left(l^{-}\right)}\right|$and $\xi$ is $\eta$ or $y$, the pseudorapidity $\left(\eta=-\log \tan \frac{\theta}{2}\right)$ or rapidity $\left(y=\frac{1}{2} \ln \frac{E+p_{z}}{E-p_{z}}\right)$ of topquarks and its semi-leptonic decay products, respectively. Reference [37] argued that since most of the charge asymmetry is concentrated at large rapidities, the statistical significance of any measurement will be enhanced, if the sample is restricted to larger rapidities. Therefore, a complementary asymmetry is defined by

$$
\begin{aligned}
& A_{t \bar{t}}\left(Y_{\text {cut }}\right)=A_{C}^{t \bar{t}}(y)=\frac{N\left(\left|y_{t}\right|>\left|y_{\bar{t}}\right|\right)-N\left(\left|y_{\bar{t}}\right|>\left|y_{t}\right|\right)}{N\left(\left|y_{t}\right|>\left|y_{\bar{t}}\right|\right)+N\left(\left|y_{\bar{t}}\right|>\left|y_{t}\right|\right)} \\
& \text { with } \frac{\left|y_{t}+y_{\bar{t}}\right|}{2}>Y_{\text {cut }} .
\end{aligned}
$$

Moreover, a kinematic cut on

$\beta_{z, t \bar{t}}=\frac{\left|p_{z}^{t}+p_{z}^{\bar{t}}\right|}{E^{t}+E^{\bar{t}}}$

can be used to enlarge the fraction of $q \bar{q}$ events, $\sigma(q \bar{q})$, contained in the total cross section, $\sigma_{\text {total }}=\sigma(q \bar{q})+\sigma(g g)$ (see, for example [69]).

These asymmetries have been measured at the $\sqrt{s}=$ $7 \mathrm{TeV}$ LHC. The SM prediction for them is around $\sim$ $10^{-2}$ [37]. Current LHC experiments are not sensitive enough to measure a non-null asymmetry, their results being compatible with the SM and zero at one standard deviation:

$$
\begin{aligned}
A_{C}^{t \bar{t}} & =0.006 \pm 0.010[54], \\
& =-0.010 \pm 0.019[55], \\
& =0.018 \pm 0.022\left(m_{t \bar{t}}>600 \mathrm{GeV}\right)[54], \\
& =0.011 \pm 0.018\left(\beta_{z, t \bar{t}}>0.6\right)[55], \\
A_{t \bar{t}}\left(Y_{\text {cut }}=0.7\right) & =0.015 \pm 0.025[54] .
\end{aligned}
$$

The leptonic asymmetries are also compatible with zero but at two standard deviations:

$$
\begin{aligned}
A_{C}^{l^{+} l^{-}} & =0.023 \pm 0.014[53], \\
& =0.009 \pm 0.012[55] .
\end{aligned}
$$

The new physics models trying to explain the deviation found at Tevatron must have the complementary check against LHC measured asymmetries.

\section{Numerical analysis}

We present the numerical analysis for fixed values of the SUSY parameters and make plots by changing one parameter at a time. However, we stress that our programs are able to perform computations for any MSSM parameter space point and they admit SLHA [94] input for easy interaction with other programs/routines. For the SM parameters ${ }^{1}$ we use those given in [1], listed in Table 1. For the SUSY parameters, we choose four different scenarios summarized in Table 2. Here $M_{1}$ and $M_{2}$ are the $U(1)$ and $S U(2)$ gaugino mass parameters, respectively, $m_{\tilde{g}}$ is the gluino mass, $A_{f}(f=t, b, \tau)$ denotes the trilinear Higgs-quark coupling, $\mu$ is the higgsino mass parameter, $M_{A}$ is the pseudoscalar mass, $\tan \beta$ is the ratio between the Higgs fields vacuum expectation values and the last six rows correspond with the soft-SUSY-breaking parameters in the squark sector. On one side, because of the comparison with previous results and for illustrative purposes, we choose a parameter set defined

\footnotetext{
1 The most recent value for the top-quark mass is $173 \pm 0.3 \pm 0.7 \mathrm{GeV}$ (value \pm stat \pm syst) [95]. This value is very close to the ones used in this work and it does not have any consequence in our results.
} 
Table 1 Parameters of the Standard Model as in [1]

\begin{tabular}{lll}
\hline Parameter & Description & Present \\
\hline$G_{F}$ & Fermi constant & $1.1663787 \times 10^{-5} \mathrm{GeV}^{-2}$ \\
$1 / \alpha_{\text {em }}\left(m_{Z}\right)$ & $\begin{array}{l}\text { Inverse of electromagnetic } \\
\text { coupling constant }\end{array}$ & 137.035999074 \\
$\alpha_{S}\left(m_{Z}\right)$ & Strong coupling constant & 0.1184 \\
$m_{\text {top }}$ & Top-quark mass & $173.5 \mathrm{GeV}$ \\
$m_{b}^{\overline{M S}}$ & Bottom-quark mass & $4.18 \mathrm{GeV}$ \\
$m_{Z}$ & Z boson mass & $91.1876 \mathrm{GeV}$ \\
$m_{W}$ & W boson mass & $80.385 \mathrm{GeV}$ \\
\hline
\end{tabular}

Table 2 SUSY parameters for the MSSM scenarios: Def [23], mod $^{-}$[96], model 100267 [97] $\left(p M S S M^{c}\right)$ and $L S$. Masses and trilinear couplings are in $\mathrm{GeV}$

\begin{tabular}{lllll}
\hline Parameter & Def & mod $^{-}$ & $p M S S M^{c}$ & $L S$ \\
\hline$M_{1}$ & 95.6 & 95.6 & 1018 & 310.9 \\
$M_{2}$ & 200 & 200 & 2462 & 650 \\
$m_{\tilde{g}}$ & 3000 & 1500 & 3368 & 3000 \\
$A_{t}$ & 1630 & -1890 & 3793 & 1700 \\
$A_{b}$ & 1630 & -1890 & -1285 & 1700 \\
$A_{\tau}$ & 1630 & -1890 & 3827 & 0 \\
$\mu$ & 300 & 200 & 1911 & 800 \\
$\tan \beta$ & 10 & 20 & 43.02 & 10 \\
$M_{A}$ & 500 & 700 & 3002 & 495.5 \\
$M_{\tilde{L}_{1,2}}$ & 1000 & 500 & 1115 & 1000 \\
$M_{\tilde{L}_{3}}$ & 1000 & 1000 & 1086 & 1000 \\
$M_{\tilde{E}_{1,2}}$ & 1000 & 500 & 2554 & 1000 \\
$M_{\tilde{E}_{3}}$ & 1000 & 1000 & 2408 & 1000 \\
$M_{\tilde{q}_{1,2}}$ & 800 & 1500 & 1100 & 1000 \\
$M_{\tilde{q}_{3}}$ & 800 & 1000 & 1624 & 700 \\
$M_{\tilde{U}_{1,2}}$ & 1000 & 1500 & 2604 & 1000 \\
$M_{\tilde{U}_{3}}$ & 1000 & 1000 & 2829 & 1000 \\
$M_{\tilde{D}_{1,2}}$ & 1000 & 1500 & 3156 & 1000 \\
$M_{\tilde{D}_{3}}$ & 1000 & 1000 & 3024 & 1000 \\
\hline & & & &
\end{tabular}

as Def [23]. This scenario has been largely explored in the above reference within a very good accuracy of the effective approximation, namely that the effective approximation provides a good description of the radiative-corrected squark partial decay widths if the gluino mass is heavier than the topsquark mass. On the other side, SUSY parameters are chosen from a modification of the $m_{\max }^{h}$ scenario as in [96] with negative trilinear couplings $\left(\bmod ^{-}\right)$, and the model 100267 from [97] $\left(p M S S M^{c}\right)$. Finally, we define a new scenario with relatively small masses for the squarks of the third generation in order to provide a scenario with top squarks capable of being produced in the next run of the LHC and compatible with the effective approximation used along this work.
We denote this parameter choice $L S$ in our numerical analysis, corresponding with a scenario with light squark. Even if we present the input parameters for all the scalar sector, we restrict ourselves in the numerical analysis to the case of third generation squarks. With these input parameters, the central values for the physical SUSY particle masses are given in Table 3.

At present the ATLAS and CMS collaborations have already put some stringent limits [98-105] on the masses of gluino and squarks, specially of the first and second generations. Our top-squark mass parameter choices are larger than the excluded ones; moreover, the exclusion limits would be loosened by allowing the existence of several decay channels for the top squark. Besides, since we are interested in the effective description of squark interactions, the gluino mass is chosen preferably large to enhance the effects of the logarithmic terms. Note also that if the gluino decay channel is open, it will be the dominant decay channel for squarks, rendering the chargino/neutralino channels phenomenologically irrelevant. Therefore our region of interest is

$m_{\tilde{g}}+m_{q}>m_{\tilde{q}}$

The analysis of the accuracy of the effective approximation was performed in [22], using the parameter set Def as an example. We have additionally checked that the same conclusions hold for the MSSM scenarios analyzed in the present work, namely that the effective approximation provides a good description of the radiative-corrected partial decay widths, if the gluino mass is heavier than the topsquark mass $\left(m_{\tilde{g}} \gtrsim 1000 \mathrm{GeV}\right)$. We have also checked that our SUSY parameters sets are compatible with present values of the Higgs-boson mass [2,3]. We use a self-coded routine containing the expressions of [106] for the computation of the Higgs-boson mass at two-loop level. The results for the mass of the lightest CP-even Higgs-boson mass for each scenario are also included in Table 3.

Our aim is the phenomenological analysis of the MSSM contributions to the top-quark charge asymmetries and topquark polarization at the LHC. In particular, we concentrate in the contributions coming from the decays of the top squarks $\left(\tilde{t}_{1}\right.$ and $\left.\tilde{t}_{2}\right)$ into top quarks plus missing energy, both at tree level and at one-loop order. Figure 1 shows the generic Feynman diagrams contributing the most to $\sigma(q \bar{q} \rightarrow(q \chi)(\bar{q} \chi))$. Here $\tilde{f}$ denotes the squarks of the first and the second generations. The left panel of Fig. 1 includes the generic double resonant diagrams $\left(\sigma\left(q \bar{q} \rightarrow \tilde{q}_{a} \tilde{q}_{a}^{*} \rightarrow\right.\right.$ $(q \chi)(\bar{q} \chi)))$ and the right panel shows the single resonant diagrams. Regarding the simulation procedure, we have generated 5 millions of events, by means of MadGraph, for each one of the four SUSY scenarios described in Table 2 and also 5 millions of events for the $t \bar{t}$ SM background, computed at NLO. 
Table 3 Masses of SUSY particles, in $\mathrm{GeV}$

\begin{tabular}{lllll}
\hline Masses & Def & mod $^{-}$ & $p$ MSSM & LS \\
\hline$m_{\tilde{g}}$ & 3000 & 1500 & 3368 & 3000 \\
$m_{\tilde{t}_{1}}$ & 718.8 & 835.7 & 1609 & 626.9 \\
$m_{\tilde{t}_{2}}$ & 1086 & 1165 & 2848 & 1074 \\
$m_{\tilde{\chi}^{0}}$ & $91.7,176.3-334.6$ & $87.9,151.4-266.4$ & $1017,1906-2469$ & $309.3,629.5-824.6$ \\
$m_{\tilde{\chi}^{+}}$ & $175.3,334.9$ & $147.5-266.8$ & 1905,2469 & $629.3,824.2$ \\
$m_{h}$ & 125.3 & 127.1 & 127.8 & 125.6 \\
\hline
\end{tabular}

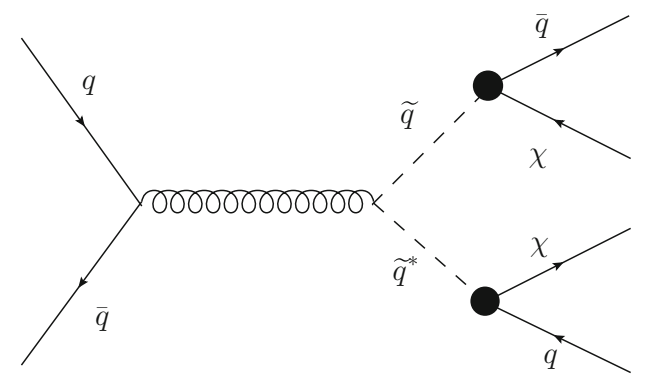

(a)

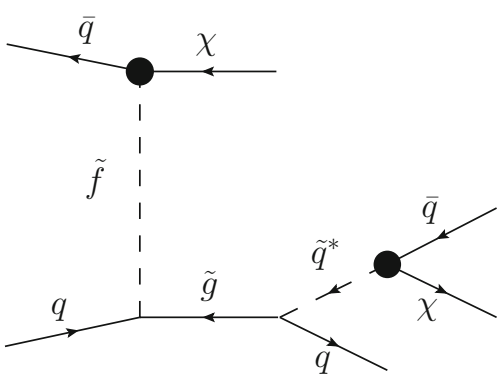

(b)
Table 4 Branching ratios of $\tilde{t}_{1,2}$ decays for different MSSM scenarios, at tree level. $\tilde{t}_{2} \rightarrow X$ stands for the sum of branching of all other possible $\tilde{t}_{2}$ decay channels

\begin{tabular}{lllll}
\hline $\operatorname{Branching}$ ratio & Def & mod $^{-}$ & $p M S S M^{c}$ & $L S$ \\
\hline $\operatorname{BR}\left(\tilde{t}_{1} \rightarrow t \tilde{\chi}_{1}^{0}\right)$ & 0.011 & 0.057 & 1 & 1 \\
$\operatorname{BR}\left(\tilde{t}_{1} \rightarrow t \tilde{\chi}_{2}^{0}\right)$ & 0.073 & 0.148 & 0 & 0 \\
$\operatorname{BR}\left(\tilde{t}_{1} \rightarrow t \tilde{\chi}_{3}^{0}\right)$ & 0.186 & 0.296 & 0 & 0 \\
$\operatorname{BR}\left(\tilde{t}_{1} \rightarrow t \tilde{\chi}_{4}^{0}\right)$ & 0.352 & 0.058 & 0 & 0 \\
$\operatorname{BR}\left(\tilde{t}_{1} \rightarrow b \tilde{\chi}_{1}^{+}\right)$ & 0.125 & 0.385 & 0 & 0 \\
$\operatorname{BR}\left(\tilde{t}_{1} \rightarrow b \tilde{\chi}_{2}^{+}\right)$ & 0.251 & 0.056 & 0 & 0 \\
$\operatorname{BR}\left(\tilde{t}_{2} \rightarrow t \tilde{\chi}_{1}^{0}\right)$ & 0.030 & 0.010 & 0.029 & 0.030 \\
$\operatorname{BR}\left(\tilde{t}_{2} \rightarrow t \tilde{\chi}_{2}^{0}\right)$ & 0.048 & 0.045 & 0.051 & 0.019 \\
$\operatorname{BR}\left(\tilde{t}_{2} \rightarrow t \tilde{\chi}_{3}^{0}\right)$ & 0.151 & 0.146 & 0.054 & 0.041 \\
$\operatorname{BR}\left(\tilde{t}_{2} \rightarrow t \tilde{\chi}_{4}^{0}\right)$ & 0.071 & 0.194 & $\sim 10^{-5}$ & 0.008 \\
$\operatorname{BR}\left(\tilde{t}_{2} \rightarrow b \tilde{\chi}_{1}^{+}\right)$ & 0.107 & 0.028 & 0.109 & 0.048 \\
$\operatorname{BR}\left(\tilde{t}_{2} \rightarrow b \tilde{\chi}_{2}^{+}\right)$ & 0.139 & 0.258 & $\sim 10^{-5}$ & 0.042 \\
$\operatorname{BR}\left(\tilde{t}_{2} \rightarrow X\right)$ & 0.454 & 0.319 & 0.757 & 0.812 \\
\hline
\end{tabular}

First of all, we have computed the top-squark partial decay widths in each scenario, focusing on reactions where both $\tilde{t}_{1}$ and $\tilde{t}_{2}$ decay into a top-quark and the lightest neutralino $\tilde{\chi}_{1}^{0}$. Results for all the branching ratios at tree level are presented in Table 4. Note that the more convenient MSSM scenarios for our purpose are $p M S S M^{c}$ and $L S$. In these two cases $\operatorname{BR}\left(\tilde{t}_{1} \rightarrow t \tilde{\chi}_{1}^{0}\right)$ is maximal, the other $\tilde{t}_{1}$ decays channels being closed. For $\tilde{t}_{2}$ all decays channels are open being $\operatorname{BR}\left(\tilde{t}_{2} \rightarrow X\right)\left(X \equiv \tilde{t}_{1} h, \tilde{t}_{1} Z^{0}, \tilde{b}_{1} W^{+}\right)$maximal. In the Def and $\mathrm{mod}^{-}$scenarios there exist other allowed decay chan- nels for the top squarks and the $\tilde{t}_{1,2} \rightarrow t \tilde{\chi}_{1}^{0}$ are suppressed. However, we choose these two other MSSM sets to have a more complete phenomenological analysis and to arrive at general conclusions.

The SUSY-QCD contributions we are interested in are

$p p \rightarrow t \bar{t} \tilde{\chi}_{1}^{0} \tilde{\chi}_{1}^{0} \rightarrow b \bar{b} l v_{l} l^{\prime} v_{l^{\prime}} \tilde{\chi}_{1}^{0} \tilde{\chi}_{1}^{0}$,

where $p$ implies $g, u, d, s, c$ initial states and $l$ resume $e$ and $\mu$ leptons. Besides, leptons in the final state appear only as product of the top-quark decay chain $t \rightarrow b W \rightarrow b l v_{l}$. We apply kinematic cuts on the transverse momentum $p_{T}$ and pseudo-rapidity $\eta$ of the final leptons and $b$ quarks, being $p_{T}>20 \mathrm{GeV}$ and $|\eta|<2.5$.

Relative deviation, $\delta$ in $\%$, of any quantity, $\mathrm{R}$, is calculated as:

$\delta=\frac{R^{\text {eff }}-R^{\text {tree }}}{R^{\text {tree }}} \times 100$.

Table 5 shows the results for the cross section $\sigma(p p \rightarrow$ $\left.b \bar{b} l l^{\prime}+E_{T}^{\text {miss }}\right)$ in pb both in the $\operatorname{SM}\left(E_{T}^{\text {miss }}=v_{l} v_{l^{\prime}}\right)$ and in the MSSM $\left(E_{T}^{\text {miss }}=v_{l} v_{l^{\prime}} \tilde{\chi}_{1}^{0} \tilde{\chi}_{1}^{0}\right)$ at the $\sqrt{s}=14 \mathrm{TeV}$ LHC. We use MadGraph for the computation. The values of the cross section are given in each scenario both at tree level and in the effective approximation. Besides, the results for the fraction of surviving events above certain cut on $p_{T}^{\text {miss }}$ defined as $p_{T}^{\text {miss }}=\sum_{i=v_{l}, \tilde{\chi}_{l}^{0}} p_{T}^{i}$ are presented in the last two columns. $\kappa$ is the fraction of events surviving a giving cut on $p_{T}^{\text {miss }}$. As expected, the cross section in all the MSSM scenarios is suppressed with respect to the SM one. The values of the MSSM cross section are between $2 \mathrm{fb}$ and $10^{-4} \mathrm{fb}$, 
Table 5 Cross section of $p p \rightarrow b \bar{b} l l^{\prime}+E_{T}^{\mathrm{miss}}$ in the SM $\left(E_{T}^{\text {miss }}=v_{l} v_{l^{\prime}}\right)$ and in the $\operatorname{MSSM}\left(E_{T}^{\mathrm{miss}}=v_{l} v_{l^{\prime}} \tilde{\chi}_{1}^{0} \tilde{\chi}_{1}^{0}\right)$ for studied SUSY scenarios at the $\sqrt{s}=14$ TeV LHC. $\kappa$ is the fraction of events surviving a giving cut on $p_{T}^{\text {miss }}$

\begin{tabular}{|c|c|c|c|c|c|c|}
\hline \multirow{2}{*}{$\begin{array}{l}\text { SM } \\
\text { MSSM }\end{array}$} & \multicolumn{2}{|c|}{$\begin{array}{l}\sigma\left(p p \rightarrow b \bar{b} l l^{\prime}+E_{T}^{\text {miss }}\right)[\mathrm{fb}] \\
18.1 \times 10^{3}\end{array}$} & \multicolumn{2}{|c|}{$\begin{array}{l}\kappa_{p_{T}^{\text {miss }}>200 \mathrm{GeV}} \\
1.02 \times 10^{-2}\end{array}$} & \multicolumn{2}{|c|}{$\begin{array}{l}\kappa_{p_{T}^{\mathrm{miss}}}>300 \mathrm{GeV} \\
1.62 \times 10^{-3}\end{array}$} \\
\hline & Tree & Eff & Tree & Eff & Tree & Eff \\
\hline Def & $2.39 \times 10^{-4}$ & $4.52 \times 10^{-4}$ & 0.76 & 0.75 & 0.55 & 0.53 \\
\hline $\bmod ^{-}$ & $2.32 \times 10^{-3}$ & $3.26 \times 10^{-3}$ & 0.79 & 0.79 & 0.59 & 0.59 \\
\hline$p M S S M^{c}$ & $2.76 \times 10^{-3}$ & $2.76 \times 10^{-3}$ & 0.81 & 0.81 & 0.62 & 0.62 \\
\hline$L S$ & 2.72 & 2.77 & 0.40 & 0.40 & 0.12 & 0.12 \\
\hline
\end{tabular}

Table 6 Signal significances for the $L S$ scenario, with $S=\sigma_{S} \mathcal{L}$ and $B=\sigma_{B} \mathcal{L}$

$L S$ signal significances

\begin{tabular}{lll}
\hline Significances & $\mathcal{L}=300 \mathrm{fb}^{-1}$ & $\mathcal{L}=1000 \mathrm{fb}^{-1}$ \\
\hline$S / B$ & 0.014 & 0.014 \\
$S / \sqrt{B}$ & 1.39 & 2.54 \\
$S / \sqrt{S+B}$ & 1.38 & 2.53 \\
$S / \sqrt{B+(0.2 B)^{2}}$ & 0.07 & 0.07 \\
\hline
\end{tabular}

depending on the SUSY parameter choice. Clearly, the most promising scenario for this analysis is $L S$, having a maximum value for the cross section of about $2 \mathrm{fb}$. The radiative corrections can be large in some scenarios. For example, the relative deviation $\delta$ between the tree-level calculation and the effective results is around $89 \%$ and $40 \%$ in the Def and $\bmod ^{-}$scenarios, respectively. However, the situation change drastically for the other two scenarios, being the above relative deviation equal to or less than $1 \%$.

We complete the analysis presenting the results for $\sqrt{s}=$ $14 \mathrm{TeV}$ and $\mathcal{L}=300 \mathrm{fb}^{-1}$, the anticipated integrated luminosity that will be delivered by LHC in its first 10 years of life [107], or $\mathcal{L}=1000 \mathrm{fb}^{-1}$ for the high-luminosity LHC (HL-LHC) [108]. The $L S$ scenario has the best signal to background relations of all the scenarios presented in this work and its expected signal significances for the highest cut on $p_{T}^{\text {miss }}$ are shown in Table 6. There are some recent works very useful to identify $K$-factors that could be applied to the MSSM cross sections: a $K$-factor of $1.25-1.3$ [109] can be estimated at NLO for top-squark production at the LHC [109-113] and a $K$-factor of 1.8 [114] at next-to-nextto-leading-logarithmic (NNLL) accuracy [114-120] over the NLO cross section, resulting in an overall $K$-factor of 1.4 , which has been considered for the calculations in Table 6 . We have also to take into account the $K$-factors for the SM which can be extracted from the well-known NNLO+NNLL QCD corrections to $t \bar{t}$ production [121-125], estimating an approximate $K$-factor of 1.1. The results for the HL-LHC in Table 6 are close to the lower limits of signal observation (around 3) and whilst they could be improved somehow if one applies some more cuts optimized to reduce the SM contribution, like a cut on the top-quark polarizations, it seems difficult to increase sufficiently the signal significances to achieve values near 5, considered as the signal discovery.

In that sense, in order to try to find scenarios with more promising signal significances, one can imagine simplified models [126-131] with only one neutralino and one stop at low energies, whose masses should be much lighter than in the four scenarios considered in this work. One example of this kind of simplified models could be a modified $L S$ scenario with $M_{1}=100 \mathrm{GeV}$ and $M_{\tilde{q}_{3}}=375 \mathrm{GeV}$. This choice of soft-SUSY-breaking parameters would give rise to a supersymmetric spectrum with $m_{\tilde{\chi}_{1}^{0}}=99.3 \mathrm{GeV}$ and $m_{\tilde{t}_{1}}=310 \mathrm{GeV}$. In this case, $\operatorname{BR}\left(\tilde{t}_{1} \rightarrow t \tilde{\chi}_{1}^{0}\right)=1$ and then we would obtain $\sigma\left(p p \rightarrow \tilde{t}_{1} \tilde{t}_{1} \rightarrow b \bar{b} l l^{\prime}+E_{T}^{\text {miss }}\right) \sim 150 \mathrm{fb}$ at $\sqrt{s}=14 \mathrm{TeV}$. If we consider a similar fraction of surviving events around $10 \%$ after applying cuts on $p_{T}^{\text {miss }}$ as in Table 5 for the $L S$ scenario, the resulting cross section would be $\sim 15 \mathrm{fb}$. Taking into account the $K$-factors mentioned above for the SM background and the MSSM scenarios, the expected signal significances for this simplified model would be $S / B \sim 0.7$ (clearly larger than $\mathcal{O}(10 \%)$ which is considered as the minimum value to have an observable signal) and $S / \sqrt{B+(0.2 B)^{2}} \sim 3.4$ for both total integrated luminosities of $300 \mathrm{fb}^{-1}$ and $1000 \mathrm{fb}^{-1}$. These significances would really improve the results of Table 6 and show what class of scenarios could be testable at the LHC performing these search strategies, which are out of the scope of this work. Anyway, the effects of the effective squark approximation presented and used here are practically negligible for this kind of simplified models, depending on the SUSY parameter space.

\subsection{Top-quark charge asymmetries}

As widely argued in the literature, $t \bar{t}$ charge asymmetries could appear in proton-proton collisions at the LHC (see for example [62] and references therein). In order to investigate them, we consider the cut-independent charge asymmetries defined by Eq. (18), which are being used in current analysis by ATLAS [54] and CMS [55]. Both experiments are reporting charge asymmetries compatible with a zero 
Table 7 Top-quark charge asymmetries as a function of the rapidity $(y)$ and the pseudo-rapidity $(\eta)$, in $\%$, for the SM and the MSSM scenarios for $\beta_{z, t \bar{t}}>0\left(\beta_{z, t \bar{t}}>0.6\right)$ at the $\sqrt{s}=14 \mathrm{TeV}$ LHC

\begin{tabular}{|c|c|c|c|c|c|c|}
\hline \multirow{3}{*}{$\begin{array}{l}\mathrm{SM} p p \rightarrow t \bar{t} \\
p p \rightarrow t \bar{t} \tilde{\chi}_{1}^{0} \tilde{\chi}_{1}^{0}\end{array}$} & \multicolumn{6}{|c|}{$\beta_{z, t \bar{t}}>0\left(\beta_{z, t \bar{t}}>0.6\right)$} \\
\hline & \multicolumn{2}{|l|}{$\begin{array}{l}100 \times A_{C}^{t \bar{t}}(y) \\
0.229(0.275)\end{array}$} & \multicolumn{2}{|l|}{$\begin{array}{l}100 \times A_{C}^{t \bar{t}}(\eta) \\
0.307(0.350)\end{array}$} & \multicolumn{2}{|l|}{$\begin{array}{l}100 \times A_{C}^{l^{+} l^{-}}(y) \\
0.195(0.214)\end{array}$} \\
\hline & Tree & Eff & Tree & Eff & Tree & Eff \\
\hline Def & $0.354(0.413)$ & $0.191(0.152)$ & $0.508(0.582)$ & $0.332(0.269)$ & $0.334(0.416)$ & $0.202(0.141)$ \\
\hline $\mathrm{mod}^{-}$ & $0.196(0.165)$ & $0.167(0.237)$ & $0.364(0.384)$ & $0.293(0.437)$ & $-0.371(-0.881)$ & $-0.316(-0.637)$ \\
\hline$p M S S M^{c}$ & $0.052(-0.069)$ & $-0.003(-0.017)$ & $0.059(-0.057)$ & $-0.001(0.018)$ & $-0.008(-0.114)$ & $-0.026(-0.122)$ \\
\hline LS & $0.013(0.033)$ & $-0.028(-0.017)$ & $0.004(-0.031)$ & $-0.050(-0.068)$ & $0.004(0.001)$ & $-0.060(-0.047)$ \\
\hline
\end{tabular}

value and consistent with the SM expectations. In Table 7 we present our results for the top-quark charge asymmetries, calculated from Eq. (18), as a function of particle rapidity $(y)$ and the pseudo-rapidity $(\eta)$ at $\sqrt{s}=14 \mathrm{TeV}$. Results are given for the SM in $p p \rightarrow t \bar{t}$ channel and for all the four SUSY scenarios defined in the previous section. It is important to remark here that the asymmetries are computed for one model at a time, with the denominators at the same order as the numerators. ${ }^{2}$ Our SM values are computed using a NLO approximation as included in MadGraph [132], they are meant for the comparison to the MSSM predictions. At this point we want to emphasize that a detail understanding of the decay processes and experimental cuts in both models will be necessary to combine NLO level computations in a consistent way. We only focused here on the impact of the effective corrections on top-quark charge asymmetries in several MSSM scenarios and we compare their results with the SM predictions. Our computations are done without any cut on the $z$-component of $t \bar{t}$-system velocity, $\beta_{z, t \bar{t}}$, and with a kinematic cut on $\beta_{z, t \bar{t}}$, requiring $\beta_{z, t \bar{t}}>0.6$ (results within brackets of Table 7), which defines a region of phase space where the physics beyond the SM effects on the asymmetry may be enhanced [69]. All the MSSM predictions, both at tree level and in the squark effective description approximation, are close to the SM ones, being the Def and $\mathrm{mod}^{-}$scenarios the most compatible ones with the SM predictions. As expected the $\beta_{z, t \bar{t}}>0.6$ cut increases the values of the asymmetries in most of the cases. The results for the top-quark charge asymmetries are also sensitive to the inclusion of radiative corrections through the analysis of the effective approximations. For the case of Def $\left(\mathrm{mod}^{-}\right)$scenario, when $\beta_{z, t \bar{t}}>0.6$, the relative deviation $\delta$ is about $-63 \%(43 \%)$ for $A_{C}^{t \bar{t}}(y),-53 \%(13 \%)$ for $A_{C}^{t \bar{t}}(\eta)$, and $-66 \%(-0.28 \%)$ for $A_{C}^{l^{+} l^{-}}(y)$. Therefore, the top-quark

2 The most precise current predictions for the cut-independent $t \bar{t}$ asymmetries in the SM at the $14 \mathrm{TeV}$ LHC are $A_{C}^{t \bar{t}}(y)=0.77 \times 10^{-2}$, $A_{C}^{t \bar{t}}(\eta)=0.59 \times 10^{-2}$ [37]. Our results are not comparable with the previous ones. asymmetries decrease strongly when radiative corrections are included in the case of the Def set but, to the contrary, in the $\mathrm{mod}^{-}$scenario the situation changes a bit increasing the values of $A_{C}^{t \bar{t}}$ whilst $A_{C}^{l^{+} l^{-}}(y)$ decreases. In the other two scenarios, $p M S S M^{c}$ and $L S$, the results of the deviations are compatible with zero. However, the radiative corrections can also be important, being $\delta-75 \%$ for $A_{C}^{t \bar{t}}(y)$ and $7 \%$ for $A_{C}^{l^{+} l^{-}}(y)$ in the $p M S S M^{c}$ scenario, and around $-100 \%$ for $A_{C}^{l^{+} l^{-}}(y)$ in the $L S$ set. As a conclusion, the behavior of the top-quark asymmetries with the inclusion of the radiative corrections is strongly dependent on the SUSY scenarios, and it must be taken into account in a phenomenological analysis.

Let us emphasize that the $t \bar{t}$ charge asymmetry is a consequence of Feynman diagram interferences. Thus, it is necessary that at least two diagrams from the $q \bar{q}$ channel contribute significantly, otherwise the asymmetry will be consistent with zero. Needless to say, all contributions from the $g g$ channel, despite they interfere with each other, are symmetric, and hence cancel when accounted in the calculation of Eq. (18).

First, we analyze the results for scenarios with the lowest values of the asymmetry: $p M S S M^{c}$ and $L S$. In these scenarios, at tree level, more than $99 \%$ of the $q \bar{q}$ contributions to the total cross section comes from the double resonant diagrams as in Fig. 1a with $\tilde{q} \rightarrow \tilde{t}_{1}$. When using the effective approximation of squark interactions, the relative weight of $q \bar{q}$ diagrams do not change and the mentioned diagram is by far kept as the leading one. Then, for these scenarios there is no chance of observing values of $A_{C}^{t \bar{t}}$ differing from zero regardless of the approximation used.

Second, the $\mathrm{mod}^{-}$scenario gives a positive asymmetry a bit larger than in the above two scenarios. In this case, the diagrams as in Fig. 1a with $\tilde{q} \rightarrow \tilde{t}_{1}$ give a contribution of $43 \%(48 \%)$ to the $q \bar{q}$ cross section at tree level (in the effective approximation). The contribution with $\tilde{q} \rightarrow \tilde{t}_{2}$ is less than $1.5 \%$. Correspondingly, $45 \%$ (42\%) of the $q \bar{q}$ contribution is absorbed by diagrams as in Fig. 1b, being $\tilde{q} \rightarrow \tilde{t}_{1}$ and $\tilde{f} \rightarrow \tilde{f}_{1,2}$ the squarks of the first and the second genera- 
Table 8 Top-quark charge asymmetries $A_{t \bar{t}}\left(Y_{\text {cut }}\right)$, in $\%$, for $Y_{\text {cut }}>0.7$ when $m_{t \bar{f}}<450 \mathrm{GeV}$ and $m_{t \bar{t}}>450 \mathrm{GeV}$ for the SM in $p p \rightarrow t \bar{t}$ and MSSM scenarios at the $\sqrt{s}=14 \mathrm{TeV}$ LHC

\begin{tabular}{|c|c|c|c|c|c|c|}
\hline \multirow{3}{*}{$\begin{array}{l}\mathrm{SM} p p \rightarrow t \bar{t} \\
p p \rightarrow t \bar{t} \tilde{\chi}_{1}^{0} \tilde{\chi}_{1}^{0}\end{array}$} & \multicolumn{6}{|c|}{$A_{t \bar{t}}\left(Y_{\text {cut }}>0.7\right) \times 100$} \\
\hline & \multicolumn{2}{|c|}{$\begin{array}{l}m_{t \bar{t}}>2 m_{t} \\
0.304\end{array}$} & \multicolumn{2}{|c|}{$\begin{array}{l}m_{t \bar{t}}<450 \mathrm{GeV} \\
0.301\end{array}$} & \multicolumn{2}{|c|}{$\begin{array}{r}m_{t \bar{t}}>450 \mathrm{GeV} \\
0.424\end{array}$} \\
\hline & Tree & Eff & Tree & Eff & Tree & Eff \\
\hline Def & 0.806 & 0.475 & 0.711 & -0.195 & 0.792 & 0.509 \\
\hline $\bmod ^{-}$ & 0.339 & 0.290 & -0.607 & -0.422 & 0.387 & 0.331 \\
\hline$p M S S M^{c}$ & -0.011 & 0.077 & 0.295 & -0.125 & -0.055 & 0.081 \\
\hline LS & 0.048 & -0.039 & -0.126 & -0.144 & 0.079 & 0.048 \\
\hline
\end{tabular}

tion. These squarks in the $\mathrm{mod}^{-}$scenario are degenerate and the physical masses are around $1.5 \mathrm{TeV}$, thus enhancing the interference and, therefore, changing $A_{C}^{t \bar{t}}$ towards values different from zero. In this scenario, the interference between $\tilde{t}_{1}$ and $\tilde{t}_{2}$ diagrams is small. Summarizing, the main interference comes from diagrams like in Fig. 1b with the first and second generation of squarks in the internal line and the lightest top-squark.

Finally, the Def scenario also has a different behavior of the relative weights of the contributions to the $q \bar{q}$ cross section. Diagrams of Fig. 1a with $\tilde{q} \rightarrow \tilde{t}_{1,2}$ give a $75 \%(81 \%)$ of the total cross section of the $q \bar{q}$ channel and split on $43 \%$ $(55 \%)$ for the $\tilde{t}_{1}$ and $32 \%(26 \%)$ for the $\tilde{t}_{2}$ diagram at tree level (in the effective calculation). In this scenario the larger branching ratio of the decay $\tilde{t}_{2} \rightarrow t \tilde{\chi}_{1}^{0}$ in comparison to the branching ratio of the $\tilde{t}_{1} \rightarrow t \tilde{\chi}_{1}^{0}$ compensates the difference of each pair production cross section, allowing the interference between each other. Diagrams as in Fig. 1b with $\tilde{f} \rightarrow \tilde{f}_{1}$ in the internal line contribute in about $15 \%(12 \%)$ to the $q \bar{q}$ channel cross section, evenly split between $\tilde{t}_{1}$ and $\tilde{t}_{2}$ and hence interfering between each other as well. The diagrams with $\tilde{f} \rightarrow \tilde{f}_{2}$ contributes less than a $0.5 \%$. Therefore, the asymmetry values arises from the interference between the diagrams as in Figs. $1 \mathrm{a}$ and $\mathrm{b}$ with both squarks, $\tilde{t}_{1}$ and $\tilde{t}_{2}$, and the lightest squark of the first and second generation. In summary, the reasons to explain asymmetry values different from zero in the MSSM are rather dependent on the SUSY parametrization chosen.

Since most of the charge asymmetries are focused on large rapidities [37], we also use the complementary definition of asymmetry on Eq. (19) in our analysis. Table 8 summarizes the results of $A_{t \bar{t}}\left(Y_{\text {cut }}\right)$ for the SM and our SUSY scenarios with $Y_{\text {cut }}=0.7$ and different cuts on $m_{t \bar{t}}{ }^{3}$ Recall that, by definition, $\left.A_{C}^{t \bar{t}}(y)\right|_{\beta_{z, t \bar{t}}>0}=A_{t \bar{t}}\left(Y_{\text {cut }}=0\right)$. We see clearly that the SM predictions are very dependent on the $m_{t \bar{t}}$ cut, which enhances almost a factor of 1.5 the value of the asymmetry

\footnotetext{
3 The current most precise prediction for $A_{t \bar{t}}\left(Y_{\text {cut }}=0.7\right)$ at the $14 \mathrm{TeV}$ LHC are: $0.75 \times 10^{-2}\left(m_{t \bar{t}}<450 \mathrm{GeV}\right)$ and $1.21 \times 10^{-2}\left(m_{t \bar{t}}>\right.$ $450 \mathrm{GeV}$ ) [37].
}

from $m_{t \bar{t}}>2 m_{t}$ to $m_{t \bar{t}}>450 \mathrm{GeV}$. $L S$ and $p M S S M^{c}$ predictions also increase with the $m_{t \bar{t}}$ cut, while Def and $\mathrm{mod}^{-}$ ones hardly change. The MSSM scenario most compatible with the SM predictions is $\mathrm{mod}^{-}$, while Def present results of the same order of magnitude but no so close to the SM ones. Clearly, for $p M S S M^{c}$ and $L S$ scenarios the results are at least one order of magnitude smaller, but also consistent within the statistical uncertainties.

In order to find kinematic regions where the top-squarkpair production via $q \bar{q}$ annihilation was comparable or even dominant over gluon fusion, and thus the charge asymmetry is capable to help in the search for SUSY, we show in Fig. 2 the behavior of the asymmetry $A_{t \bar{t}}\left(Y_{\text {cut }}\right)$ for all the four SUSY scenarios in comparison with the SM predictions, and for different values of the $m_{t \bar{t}}$ cut. Each bin of this figure contains at least $0.01 \%$ of the total number of simulated events (500 events). Large values of $Y_{\text {cut }}$ contain fewer events and are not shown. With this set up the largest statistical uncertainty is $4.5 \%$. We can conclude to the following statements for each scenario:

- Def: The tree and the effective approximation results are very similar in this scenario and both predictions are almost equal to the SM ones, specially for low values of $Y_{\text {cut }}$. The $m_{t \bar{t}}>450 \mathrm{GeV}$ cut shows similar results as the non-cut asymmetry.

- $\bmod ^{-}$: In this scenario there is no difference between $m_{t \bar{t}}>2 m_{t}$ and $m_{t \bar{t}}>450 \mathrm{GeV}$ cuts. In both cases and for low values of $Y_{\text {cut }}$, the predicted asymmetry is close to the SM ones. For $Y_{\text {cut }}>1.5$, the asymmetry becomes negative and could be distinguishable from the SM prediction. Again, the results with $m_{t \bar{t}}<450 \mathrm{GeV}$ are very different from the other two cuts, and we obtain very different results for the tree and the effective approximations, with the predictions of the latter very similar to the SM results.

- $p M S S M^{c}$ : All the predictions in this scenario, in both tree and effective approximations and for low values of $Y_{\text {cut }}$, are compatible with no asymmetries. The best region in order to try to distinguish this scenario from the SM is $1.5<Y_{\text {cut }}<2.2$, with $m_{t \bar{t}}<450 \mathrm{GeV}$, in which the 

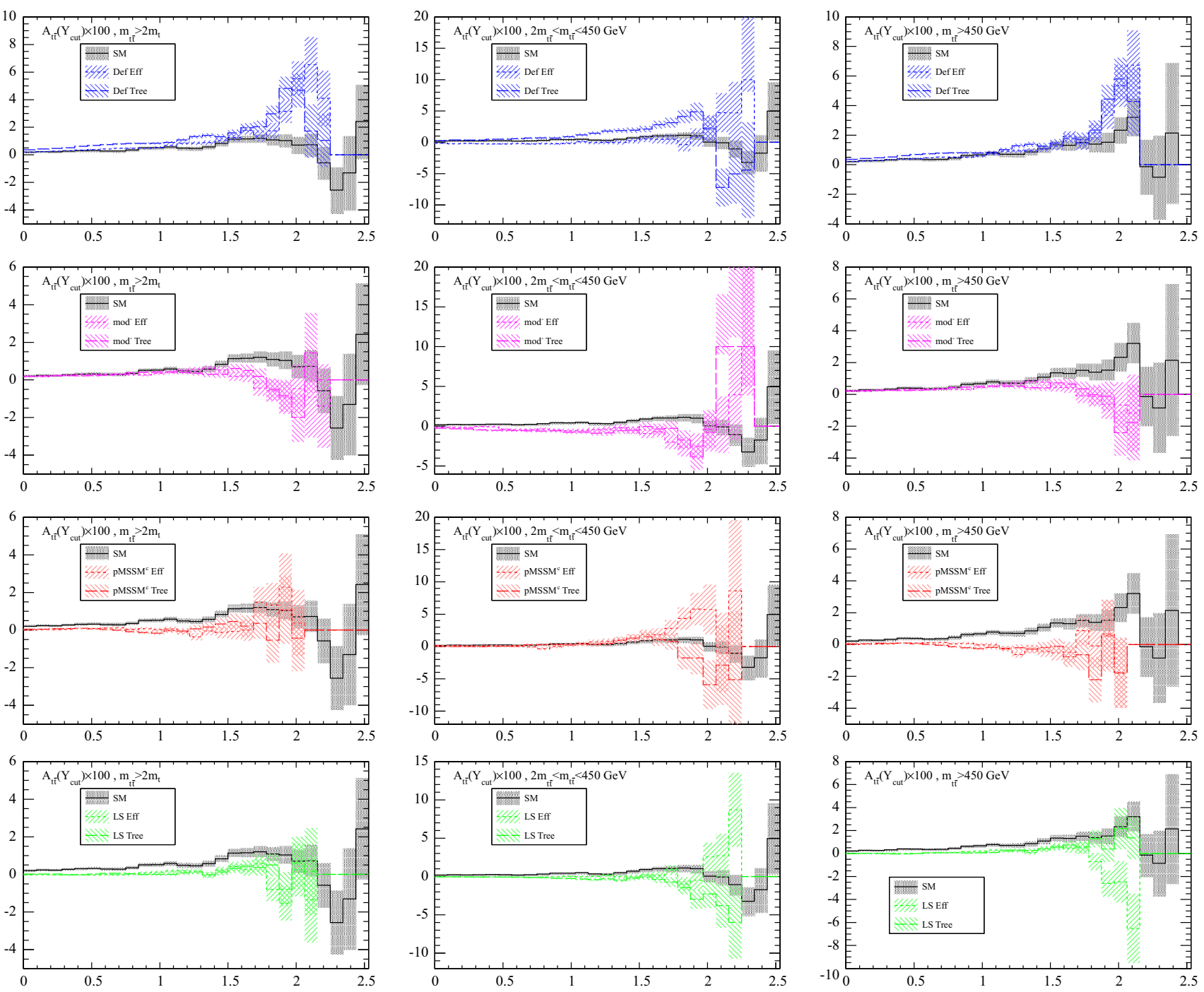

(a)

(b)

(c)

Fig. $2 A_{t \bar{t}}(Y)$ vs. $Y_{\text {cut }}$ for the SM and all SUSY scenarios: a in the whole range of $m_{t \bar{t}}$, b for $m_{t \bar{t}}<450 \mathrm{GeV}$ and $\mathbf{c}$ for $m_{t \bar{t}}>450 \mathrm{GeV}$ at the $\sqrt{s}=14 \mathrm{TeV}$ LHC

effective description provides a much larger asymmetry than the SM one whilst the tree-level results are also large but negative.

- $L S$ : For low values of $Y_{\text {cut }}$, this scenario is also compatible with no asymmetries, while in the effective approximation and for large values of $Y_{\text {cut }}$ the charge asymmetry becomes negative for $m_{t \bar{t}}>2 m_{t}$ and $m_{t \bar{t}}>450 \mathrm{GeV}$. The use of a cut in $m_{t \bar{t}}$ also enhances the size of the asymmetry for $Y_{\text {cut }}$ larger than 2 . On the other hand, the behavior of the asymmetry is very different if we consider the tree-level results. In this case, the $m_{t \bar{t}}$ cut does not help and it is very difficult to differentiate the treelevel results of $L S$ from the SM predictions.

The main conclusion of this section is that all the SUSY predictions of $A_{\mathrm{C}}$ are compatible with the SM ones. For low values of $Y_{\text {cut }}$, it is hard to distinguish between the SM and the SUSY results. On the other hand, for values of $Y_{\text {cut }}>$ 1.5 the MSSM predictions are very different from the SM ones. However, the statistical uncertainties in these cases are so large that do not allow us to draw any conclusion. In other words, we cannot make use of these $A_{\mathrm{C}}$ results in order to discriminate between the SUSY scenarios proposed along this work. Fortunately, the study of top-quark polarizations may provide additional information, useful to differentiate between scenarios as we will see in the next section.

\subsection{Top-quark polarization}

The longitudinal polarization of the top quarks coming from top-squark decays into neutralinos may supply information 
Table 9 Polarization of top quarks, $\mathcal{P}_{t}$, coming from $\tilde{t}_{1} \rightarrow t \tilde{\chi}_{1}^{0}$ decays for the MSSM scenarios with $\tan \beta=10,30,50$

\begin{tabular}{|c|c|c|c|c|c|c|}
\hline \multirow{2}{*}{$\begin{array}{l}\mathcal{P}_{t} \\
\tilde{t}_{1} \rightarrow t \tilde{\chi}_{1}^{0}\end{array}$} & \multicolumn{2}{|c|}{$\tan \beta=10$} & \multicolumn{2}{|c|}{$\tan \beta=30$} & \multicolumn{2}{|c|}{$\tan \beta=50$} \\
\hline & Tree & Eff & Tree & Eff & Tree & Eff \\
\hline Def & 0.17 & 0.39 & 0.27 & 0.44 & 0.29 & 0.44 \\
\hline $\bmod ^{-}$ & 0.83 & 0.87 & 0.89 & 0.93 & 0.90 & 0.94 \\
\hline$p M S S M^{c}$ & -0.98 & -0.95 & -0.96 & -0.93 & -0.96 & -0.92 \\
\hline$L S$ & 0.32 & 0.44 & 0.43 & 0.52 & 0.44 & 0.53 \\
\hline
\end{tabular}

as regards the SUSY scenario under study [133]. As is well known, it also differs from the unpolarized SM pair-produced top quarks. We investigate in this work if the inclusion of the radiative corrections to quark-squark-gaugino couplings [22] may change the final polarization state of the top-quarks in the SUSY framework.

First of all, we show in Table 9 the expected longitudinal top-quark polarization for each SUSY scenario, calculated with Eq. (7). As explained in $[45,48]$, the value and the sign of polarization of tops coming from $\tilde{t}_{1,2} \rightarrow t \tilde{\chi}_{1}^{0}$ decays depend on the mixing of the top-squark sector, the nature of the neutralino and the mass difference between the top-squark and the neutralino. In all the SUSY scenarios evaluated in this work, $\tilde{\chi}_{1}^{0}$ is a pure bino-like neutralino. Such bino-like neutralino couples stronger to the right-handed (RH) than to the left-handed (LH) components of the top-squark, enhancing positive values of the polarization even though the LH component of the top-squark was larger. Anyhow, whenever the LH (RH) component of the top-squark is overwhelmingly above the $\mathrm{RH}(\mathrm{LH})$ one, and the $f_{2}$ factor in Eq. (7) vanishes, ${ }^{4}$ the polarization will have values of $\mathcal{P}_{t}=-1(+1)$.

In the $p M S S M^{c}$ scenario, the lightest top-squark $\tilde{t}_{1}$ is mostly LH and therefore we obtain a top polarization very close to -1 , as shown in Table 9. The $\bmod ^{-}$scenario represents a parametrization where the mixing in the top-squark sector is maximal and the large mass difference between the top-squark and the decay products induces $f_{2} \rightarrow 0$. Due to the fact that the bino-like neutralino couples stronger to the top-squark RH component than to the $\mathrm{LH}$ one and $f_{2} \rightarrow 0$, we obtain large values of the polarization, $\sim 0.8$, in agreement with results in [45] (see Fig. 3 therein). In Def and $L S$ scenarios the top-squark mixing has similar and large $\mathrm{LH}$ components but with an important RH components which contribute more to the top-quark polarization than the $\mathrm{LH}$ ones. In these scenarios the values of the $\mathrm{LH}$ and $\mathrm{RH}$ couplings (which are of the same sign) in Eq. (7) are rather similar. The increase of the polarization values for the $L S$ scenario

\footnotetext{
4 This factor does not trivially vanishes for large mass difference between the top-squark and its decays products. It also needs a relatively small mass for the gaugino to be negligible.
}

with respect to Def scenario lies on the difference between the value of $f_{2}$ in Eq. (7). In the Def scenario $f_{2} \rightarrow 0$ meanwhile in the $L S$ scenario $f_{2} \approx 0.5$. The denominator of Eq. (7) for the $L S$ scenario is smaller than for the Def scenario and then the polarization is larger for the former than for the latter. A small variation on the values of $\mathcal{P}_{t}$ can also be appreciated as a function of $\tan \beta$, since this parameter slightly modifies both the top-squark and the neutralino mixings, and consequently the LH and RH components of top squarks and the nature of the lightest neutralino, $\tilde{\chi}_{1}^{0}$.

These changes would also be reflected on the final leptons angular distribution and hopefully measured through the asymmetries defined as in Eqs. (15) and (13). Table 10 displays the predictions for the angular asymmetries, $A_{\phi_{l}}$ and $A_{\theta_{l}}$, for the SM and the four MSSM scenarios studied here. We have checked that our SM results agree with [83] under the same cut conditions. In the last column of this table we also include the values of $\mathcal{P}_{t}$ for the decay $\tilde{t}_{1} \rightarrow t \tilde{\chi}_{1}^{0}$ with the SUSY parameters as in Table 2 . As already discussed, polarization values different from 0 translate into sharply peaked lepton distribution in $\phi_{l}$-towards 0 and $2 \pi$ - and $\theta_{l}$-towards 0 - as can be observed in the left column of Fig. 3 where the normalized distribution of both variables are presented for the SM and all MSSM parametrizations. As expected, when comparing to SM results larger asymmetries values for all MSSM scenarios are obtained.

Figure 4 shows the normalized top-quark boost distribution, $\beta_{t}$ as in Eq. (11), for the SM and all MSSM scenarios. Note that Figs. 3 and 4 only show the result in the effective approximation. The tree-level results are not presented here because they are indistinguishable in the plots. Top-quark boost distributions are presented for $\beta_{t}>0.4$ as it reflects the characteristics of more than $99 \%$ of the top-quark population for any model or parametrization. We clearly see how in Def, mod $^{-}$, and $p M S S M^{c}$ scenarios a large fractions of top quarks ( $\gtrsim 98 \%$ ) is emitted with $\beta_{t}>0.8$, supporting further the sizable values of the asymmetries obtained for those scenarios. The highly boosted top-quark populations smear the possible changes in the asymmetries due to $\mathcal{P}_{t}$ variations. For example, in the $D e f$ scenario, a change in $\mathcal{P}_{t}$ of $+130 \%$ due to radiative corrections translates into a tiny $\sim 0.4 \%, \sim 1.06 \%$ change in $A_{\phi_{l}}, A_{\theta_{l}}$, respectively. Opposite, the $L S$ scenario shows the less boosted top-quark populations (Fig. 4), and it has the smallest asymmetries (Table 10), hence the asymmetries are more sensitive to $\mathcal{P}_{t}$ variations. A $\mathcal{P}_{t}$ change of $37 \%$ due to radiative corrections translates into a $1.3 \%$, $4.5 \%$ change in $A_{\phi_{l}}, A_{\theta_{l}}$, respectively. Even though the $L S$ scenario gives the lowest asymmetries between our SUSY scenarios, the predictions in the effective approximation are a $40 \%$ and $88 \%$ larger than the SM values for $A_{\phi_{l}}$ and $A_{\theta_{l}}$, respectively.

To asseses the effects of the radiative corrections, we define the ratio of the corrected-to-tree-level distributions, 

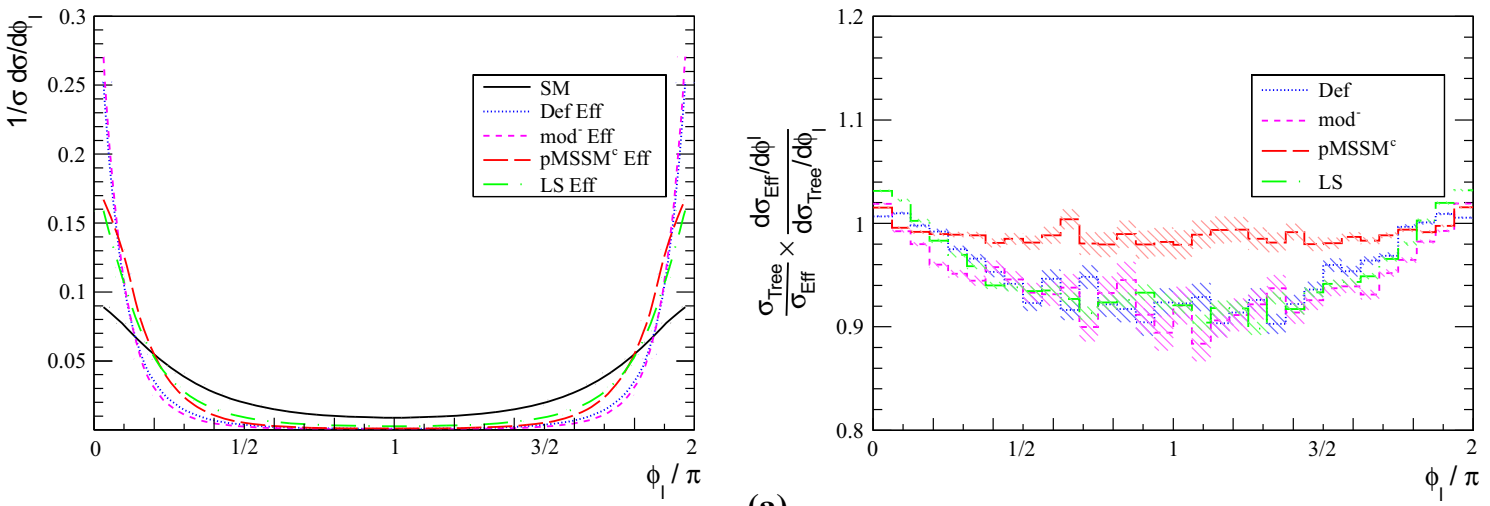

(a)
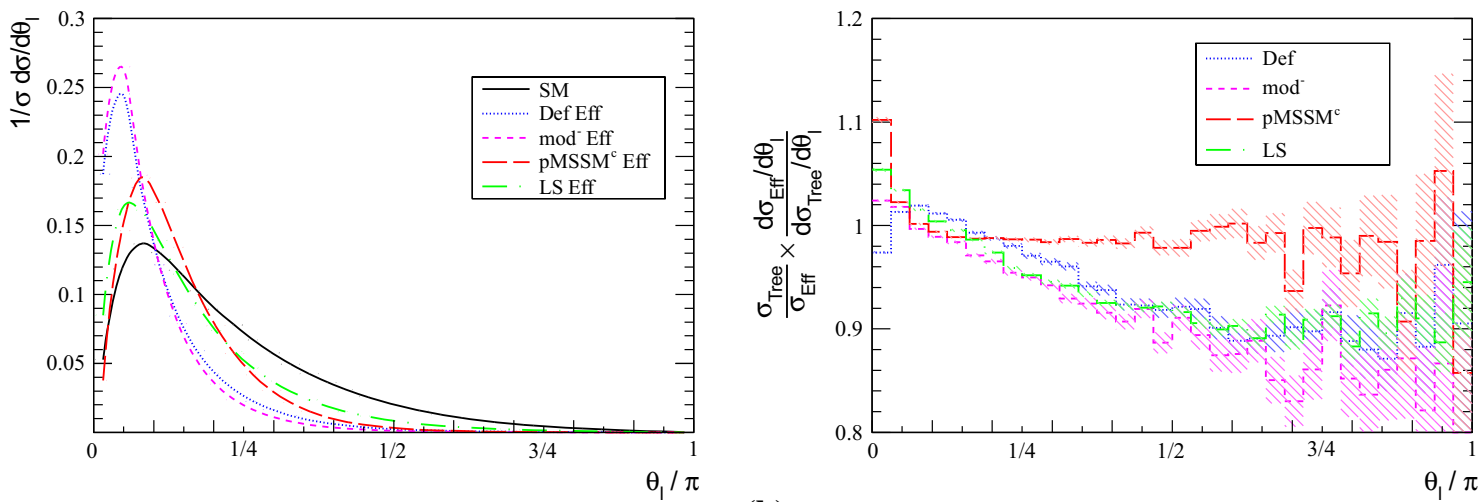

(b)

Fig. 3 Normalized cross section distribution as a function of a the azimuthal angle $\phi_{l}$ and $\mathbf{b} \theta_{l}$ of the decay lepton for the SM and all SUSY scenarios studied at $\sqrt{s}=14 \mathrm{TeV}$. The right panels show the factor $d K_{\mathrm{SUSY}} / d x$, Eq. (24)

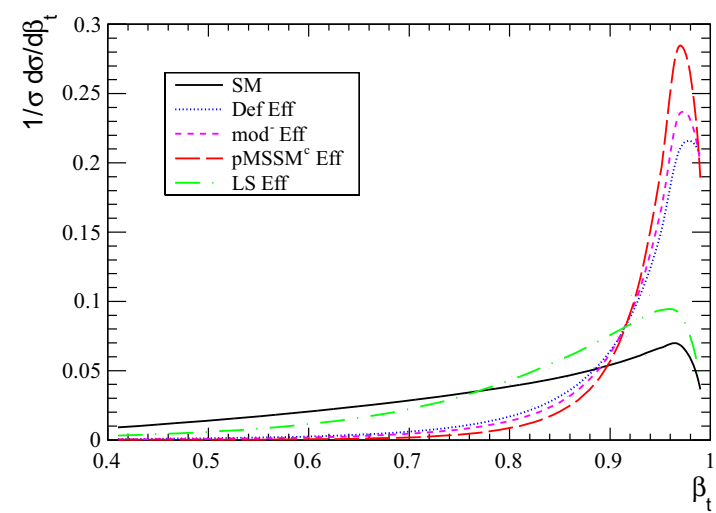

Fig. 4 As in Fig. 3 but as a function of $\beta_{t}$

$\frac{d K_{\text {SUSY }}}{d x}=\frac{\sigma_{\text {tree }}}{\sigma_{\text {eff }}} \frac{d \sigma_{\text {eff }} / d x}{d \sigma_{\text {tree }} / d x}$,

where $x$ is a distribution variable. The right panels of Figs. 3 and 4 show the effects of the radiative corrections on the $\phi_{l}, \theta_{l}$, and $\beta_{t}$ distributions. The radiative corrections change slightly the angular distributions in the $\phi_{l} \sim \pi$ and $\theta_{l} \sim \pi$ regions, up to a $10 \%$ for $\phi_{l}$ and a $20 \%$ for $\theta_{l}$. The exception is the $p M S S M^{c}$ scenario whose $d K_{\mathrm{SUSY}} / d \phi_{l}$ and

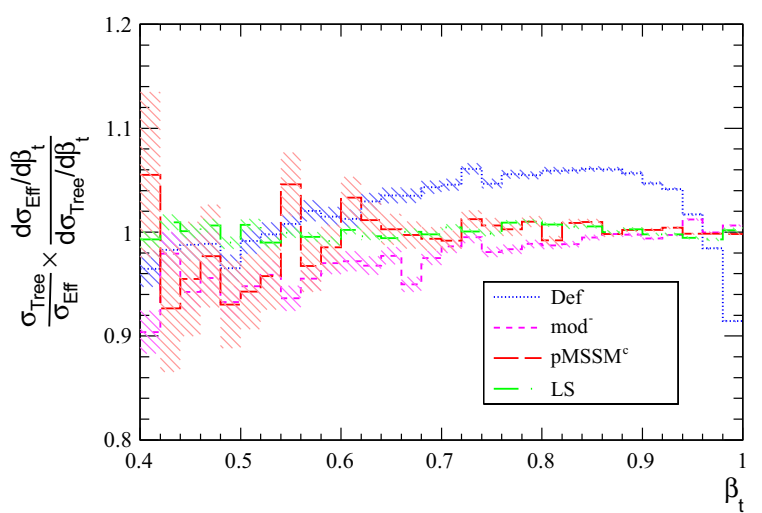

$d K_{\mathrm{SUSY}} / d \theta_{l}$ (24) factors are $\sim 1$ in the whole range. As for the $\beta_{t}$ distribution the $d K_{\mathrm{SUSY}} / d \beta_{t}$ (24) factor is $\sim 1$ for all scenarios, except $D e f$. For Def $d K_{\mathrm{SUSY}} / d \beta_{t}$ is $\sim 1.05$ for $0.7<\beta_{t}<0.9$ and 0.9 at the largest $\beta_{t}$ bin.

As mentioned in [48] and references therein, the measurements of lepton angular asymmetries in the highly boosted top-quark scenarios is a challenge at the LHC. Thus, distribution of $u$ and $z$ variables, defined as in Eqs. (16)-(17), may serve as a better discriminator for new physics scenarios 
Table 10 Lepton asymmetries for the SM in $p p \rightarrow t \bar{t}$-first row- and in the MSSM in $p p \rightarrow t \bar{t} \tilde{\chi}_{1}^{0} \tilde{\chi}_{1}^{0}$ at the $\sqrt{s}=14 \mathrm{TeV}$ LHC

\begin{tabular}{|c|c|c|c|c|c|c|}
\hline \multirow{2}{*}{$\begin{array}{l}\mathrm{SM} p p \rightarrow t \bar{t} \\
p p \rightarrow t \bar{t} \tilde{\chi}_{1}^{0} \tilde{\chi}_{1}^{0}\end{array}$} & \multicolumn{2}{|l|}{$\begin{array}{l}A_{\phi_{l}} \\
0.6165\end{array}$} & \multicolumn{2}{|l|}{$\begin{array}{l}A_{\theta_{l}} \\
0.3134\end{array}$} & \multicolumn{2}{|l|}{$\begin{array}{l}\mathcal{P}_{t} \\
0.0\end{array}$} \\
\hline & Tree & Eff & Tree & Eff & Tree & Eff \\
\hline Def & 0.9483 & 0.9522 & 0.8214 & 0.8301 & 0.17 & 0.39 \\
\hline $\bmod ^{-}$ & 0.9689 & 0.9713 & 0.8824 & 0.8903 & 0.87 & 0.91 \\
\hline$p M S S M^{c}$ & 0.9381 & 0.9390 & 0.7174 & 0.7214 & -0.96 & -0.93 \\
\hline LS & 0.8514 & 0.8626 & 0.5636 & 0.5892 & 0.32 & 0.44 \\
\hline
\end{tabular}

enhancing top-quark transverse polarization. Figures 5 and 6 show the normalized distribution of the $u$ and $z$ variables for the SM and the four MSSM scenarios in the effective description approximation of squark interactions, (a) without any cut on $\beta_{t}$ and (b) requiring a kinematic cut $\beta_{t}>0.8$. We have checked that larger kinematic cuts do not change the distribution. In agreement with [48], the $p M S S M^{c}$ scenario, with the negative polarization, show the largest difference with respect the SM with a shift on the $u$ distribution about
0.1 units toward lower values. The positively polarized topquark scenarios $\left(L S, \mathrm{mod}^{-}\right.$, and Def) differ from the SM predictions only slightly in the range $0.7<u<0.95$, a situation that worsens when the cut on $\beta_{t}$ is required. For the $z$ distributions, the positively polarized top-quark scenarios are hard to distinguish between each other independently of the $\beta_{t}$ cut, their shape resembles the SM one as the $\beta_{t}$-cut is increased. Regarding to the radiative corrections, the $u$ and $z$ distributions are hardly affected. The ratio between the effective and tree-level distributions differ on a couple of units of percent in boundary values of the variables: $u, z \approx 0$ and $u, z \approx 1$. This ratio is close to 1 in the rest of the numerical intervals of these two parameters. Therefore, these observables are unaffected by the higher order corrections in the quark-squark-chargino/neutralino interactions.

Going back to the discussion as regards scenarios with more promising signal significances (see paragraph just above Sect. 4.1), we stress that the distribution of the energy ratio $z$ does not change significantly, in the context of the radiative corrections to the quark-squark-chargino/ neutralino interactions.

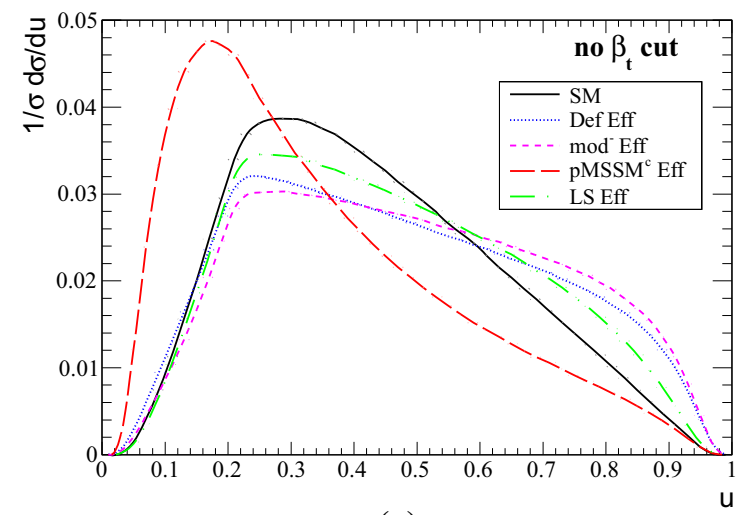

(a)

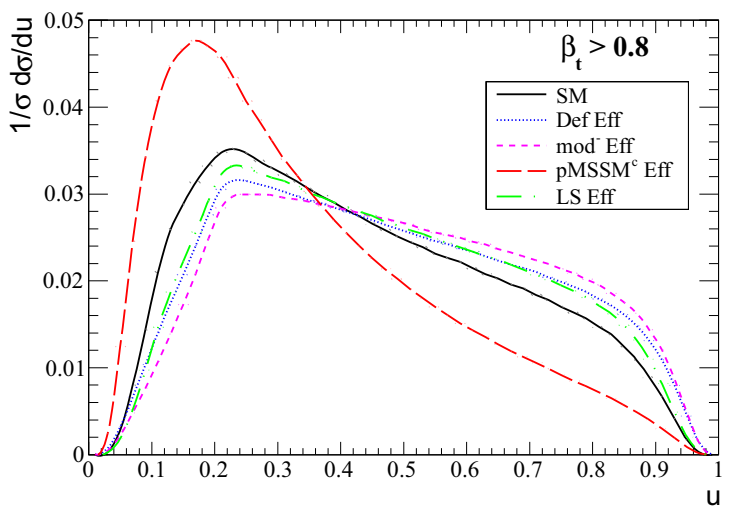

(b)

Fig. 5 Distribution of the energy ratio $u$ (a) without cut on $\beta_{t}$, and (b) for $\beta_{t}>0.8$ for the SM and all SUSY scenarios at $\sqrt{s}=14$ TeV

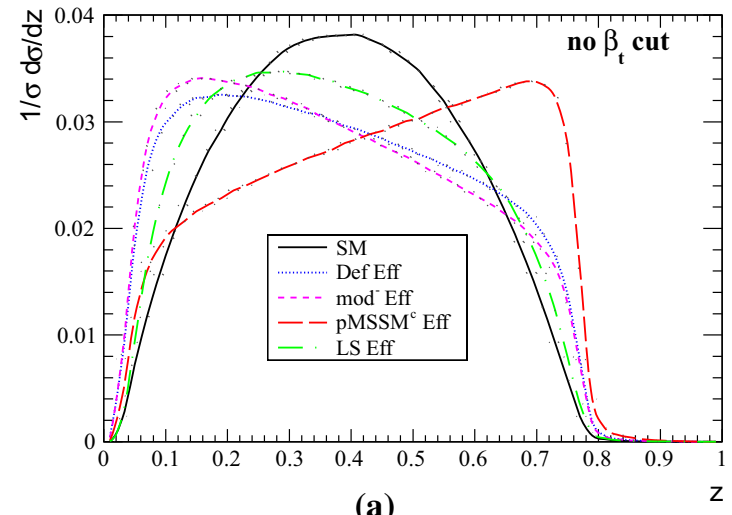

(a)

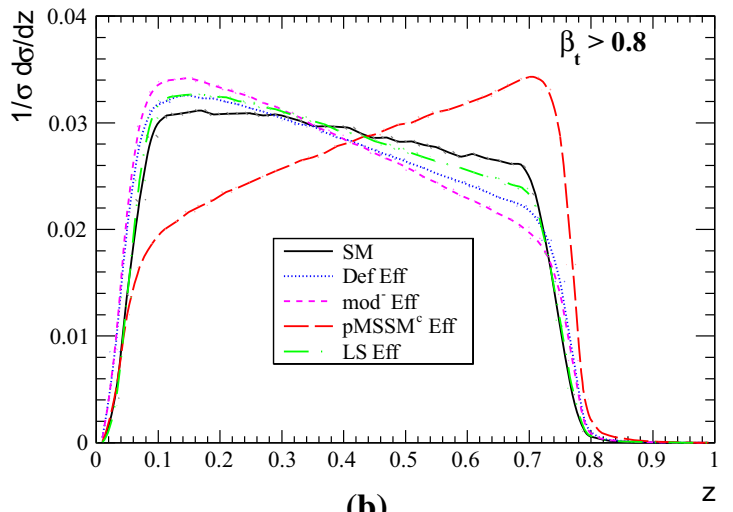

(b)

Fig. 6 As in Fig. 5 but as a function of $z$ 


\section{Conclusions}

We have computed and analyzed the top-quark polarizations and $t \bar{t}$ charge asymmetries, induced by top-squark pair production at the LHC and the subsequent decays $\tilde{t}_{1} \rightarrow t \tilde{\chi}_{1}^{0}$. The computations have been performed with MadGraph, including the effective description of squark interactions with charginos and neutralinos for the MSSM case. We have considered four different SUSY scenarios, as presented in Table 2, and we have focused on the effects of the effective approximation of squark interactions in $t \bar{t}$ charge asymmetries and top-quark polarization observables as defined in Sect. 3. We compare the results with the SM expectations, computed using the leading NLO QCD corrections as included in MadGraph 5.

The SUSY contributions to top-quark charge asymmetries, Eqs. (18) and (19), are strongly dependent on the SUSY scenarios, change significantly with the inclusion of the radiative corrections and our estimations are of the same order of the SM predictions (Tables 7, 8). Using a kinematic cut $Y_{\text {cut }}>1.5$ enhances the SUSY contributions. Unfortunately, they are still smaller than current experimental sensitivity.

For the top-quark polarization studies, the radiative corrections to the quark-squark-chargino/neutralino vertices increase the value of the polarization of the top-quarks coming from the squark decay, compared to the tree-level prediction (Table 9). We have discussed in detail the behavior of the top-quark polarization in all the SUSY scenarios studied in this work, in which $\tilde{\chi}_{1}^{0}$ is a pure bino-like neutralino. The top-quark polarization varies between -1 and 1 , depending also on the mixing of the top-squark sector and the mass difference between the top-squark and the neutralino. The changes in the top-quark polarization induce a change in the distributions and asymmetries of the lepton coming from topquark decays (Table 10). Because the top-quarks are highly boosted (Fig. 4), the polarization effects are smeared in the final distribution. The effects are more visible in scenarios with less boosted top quarks $(L S)$.

The discrimination power of the $u$ and $z$ variables, Eqs. (16) and (17), of MSSM scenarios against SM was also tested. The scenario with negative polarization of the top quarks, $p M S S M^{c}$, shows the maximal discrimination power against the SM independently of the kinematic cut applied on the top-quark boost. In this analysis we find that the inclusion of the higher order corrections through the effective description of the squark interactions does not change strongly the $u$ and $z$ distributions obtained at leading order (Figs. 5, 6).

We have found strong effects of the radiative corrections on the top-quark polarization. One would welcome new topquark observables and strategies aimed at analyzing topquark polarization.
Acknowledgments A. A. have been supported by the Spanish MICINN grant FPA2012-35453 and a SANTANDER Scholarship Program for Latinoamerican students. E. A. and S. P. have been supported by the Spanish DGIID-DGA grant 2013-E24/2 and the Spanish MICINN grant FPA2012-35453. S. P. is also supported by DURSI 2009-SGR-502. The Spanish Consolider-Ingenio 2010 Program CPAN (CSD2007-00042) has also supported this work, and E. A. particularly acknowledges this funding. S. P. thanks Germán Rodrigo for useful discussions and J. Guasch for a carefully reading of the manuscript.

Open Access This article is distributed under the terms of the Creative Commons Attribution License which permits any use, distribution, and reproduction in any medium, provided the original author(s) and the source are credited.

Funded by $\mathrm{SCOAP}^{3}$ / License Version CC BY 4.0.

\section{References}

1. J. Beringer et al., Particle Data Group Collaboration. Phys. Rev. D 86, 010001 (2012)

2. G. Aad et al., ATLAS Collaboration. Phys. Lett. B 716, 1 (2012). arXiv:1207.7214 [hep-ex]

3. S. Chatrchyan et al., CMS Collaboration. Phys. Lett. B 716, 30 (2012). arXiv:1207.7235 [hep-ex]

4. H.E. Haber, in The Woodlands 1993, Proceedings, Recent advances in the superworld 27-51, and Calif. Univ. Santa CruzSCIPP 93-022 (93/07, rec. Aug.) 26 p. hep-ph/9308209

5. H.P. Nilles, Phys. Rept. 110, 1 (1984)

6. H.E. Haber, G.L. Kane, Phys. Rept. 117, 75 (1985)

7. A.B. Lahanas, D.V. Nanopoulos, Phys. Rept. 145, 1 (1987)

8. S. Ferrara (ed.), Supersymmetry (North-Holland/World Scientific, Amsterdam/Singapore, 1987)

9. D.J.H. Chung, L.L. Everett, G.L. Kane, S.F. King, J.D. Lykken, L.-T. Wang, Phys. Rept. 407, 1 (2005). hep-ph/0312378

10. M.S. Carena, H.E. Haber, Prog. Part. Nucl. Phys. 50, 63 (2003). hep-ph/0208209

11. T. Hahn, S. Heinemeyer, W. Hollik, H. Rzehak, G. Weiglein, Phys. Rev. Lett. 112, 141801 (2014). arXiv:1312.4937 [hep-ph]

12. A. Bartl, W. Majerotto, W. Porod, Z. Phys. C 64, 499 (1994) [Erratum-ibid. C 68518 (1995)]

13. K.-i Hikasa, M. Kobayashi, Phys. Rev. D 36, 724 (1987)

14. T. Han, K.-i. Hikasa, J.M. Yang, X.-m. Zhang, Phys. Rev. D 70, 055001 (2004). hep-ph/0312129

15. F. del Aguila et al., Eur. Phys. J. C 57, 183 (2008). arXiv:0801.1800 [hep-ph]

16. W. Porod, T. Wohrmann, Phys. Rev. D 55, 2907 (1997). [Erratumibid. D 67, 059902 (2003)]. hep-ph/9608472

17. W. Porod, Phys. Rev. D 59, 095009 (1999). hep-ph/9812230

18. C. Boehm, A. Djouadi, Y. Mambrini, Phys. Rev. D 61, 095006 (2000). hep-ph/9907428

19. A. Djouadi, Y. Mambrini, Phys. Lett. B 493, 120 (2000). hep-ph/0007174

20. S.P. Das, A. Datta, M. Guchait, Phys. Rev. D 65, 095006 (2002). hep-ph/0112182

21. A. Djouadi, Y. Mambrini, Phys. Rev. D 63, 115005 (2001). hep-ph/0011364

22. J. Guasch, S. Peñaranda, R. Sanchez-Florit, JHEP 0904, 016 (2009). arXiv:0812.1114 [hep-ph]

23. A. Abrahantes, J. Guasch, S. Peñaranda, R. Sanchez-Florit, Eur. Phys. J. C 73, 2368 (2013). arXiv:1209.5214 [hep-ph]

24. A. Abrahantes, J. Guasch, S. Peñaranda, R. Sanchez-Florit. http://www.ffn.ub.es/guasch/progs/sqeffective/sqeffective.html

25. J. Alwall et al., JHEP 0709, 028 (2007). arXiv:0706.2334 [hep-ph] 
26. J. Alwall, M. Herquet, F. Maltoni, O. Mattelaer, T. Stelzer, JHEP 1106, 128 (2011). arXiv:1106.0522 [hep-ph]

27. G.-C. Cho et al., Phys. Rev. D 73, 054002 (2006). hep-ph/0601063

28. F. Abe et al. [CDF Collaboration], Phys. Rev. Lett. 74, 2626 (1995). hep-ex/9503002

29. S. Abachi et al. [D0 Collaboration], Phys. Rev. Lett. 74, 2632 (1995). hep-ex/9503003

30. J.H. Kuhn, G. Rodrigo, Phys. Rev. Lett. 81, 49 (1998). hep-ph/9802268

31. G. Rodrigo, J.H. Kuhn, in $Q C D$ and high energy hadronic interactions (Les Arcs, 1998), pp. 181-182

32. J.H. Kuhn, G. Rodrigo, Phys. Rev. D 59, 054017 (1999). hep-ph/9807420

33. J.H. Kuhn, G. Rodrigo, in High energy physics, vol. 2, pp. 11171120, Vancouver, 1998

34. V. Ahrens, A. Ferroglia, M. Neubert, B.D. Pecjak, L.L. Yang, Phys. Rev. D 84, 074004 (2011). arXiv:1106.6051 [hep-ph]

35. O. Antunano, J.H. Kuhn, G. Rodrigo, Phys. Rev. D 77, 014003 (2008). arXiv:0709.1652 [hep-ph]

36. W. Hollik, D. Pagani, Phys. Rev. D 84, 093003 (2011). arXiv:1107.2606 [hep-ph]

37. J.H. Kuhn, G. Rodrigo, JHEP 1201, 063 (2012). arXiv:1109.6830 [hep-ph]

38. T.A. Aaltonen et al., CDF Collaboration. Phys. Rev. D 88, 072003 (2013). arXiv:1308.1120 [hep-ex]

39. V.M. Abazov et al., D0 Collaboration. Phys. Rev. D 88, 112002 (2013). arXiv:1308.6690 [hep-ex]

40. V.M. Abazov et al. [D0 Collaboration], Phys. Rev. D 90(7), 072001 (2014). arXiv:1403.1294 [hep-ex]

41. T.A. Aaltonen et al., CDF Collaboration. Phys. Rev. Lett. 113, 042001 (2014). arXiv:1404.3698 [hep-ex]

42. E. Boos, H.U. Martyn, G.A. Moortgat-Pick, M. Sachwitz, A. Sherstnev, P.M. Zerwas, Eur. Phys. J. C 30, 395 (2003). hep-ph/0303110

43. M. Perelstein, A. Weiler, JHEP 0903, 141 (2009). arXiv:0811.1024 [hep-ph]

44. B. Bhattacherjee, S.K. Mandal, M. Nojiri, JHEP 1303, 105 (2013). arXiv:1211.7261 [hep-ph]

45. G. Belanger, R.M. Godbole, L. Hartgring, I. Niessen, JHEP 1305, 167 (2013). arXiv:1212.3526

46. G. Aad et al., ATLAS Collaboration. Phys. Rev. Lett. 111, 232002 (2013). arXiv:1307.6511 [hep-ex]

47. S. Chatrchyan et al., CMS Collaboration. Phys. Rev. Lett. 112, 182001 (2014). arXiv:1311.3924 [hep-ex]

48. G. Belanger, R.M. Godbole, S. Kraml, S. Kulkarni. arXiv:1304.2987 [hep-ph]

49. T. Aaltonen et al., CDF Collaboration. Phys. Rev. D 87, 092002 (2013). arXiv:1211.1003 [hep-ex]

50. T. Aaltonen et al., CDF Collaboration. Phys. Rev. Lett. 111, 182002 (2013). arXiv:1306.2357 [hep-ex]

51. V.M. Abazov et al. [D0 Collaboration], Phys. Rev. D 90(7), 072011 (2014). arXiv:1405.0421 [hep-ex]

52. W. Bernreuther, Z.-G. Si, Phys. Rev. D 86, 034026 (2012). arXiv: 1205.6580 [hep-ph]

53. [ATLAS Collaboration], ATLAS-CONF-2012-057

54. G. Aad et al., ATLAS Collaboration. JHEP 1402, 107 (2014). arXiv:1311.6724 [hep-ex]

55. S. Chatrchyan et al., CMS Collaboration. JHEP 1404, 191 (2014). arXiv: 1402.3803 [hep-ex]

56. J.A. Aguilar-Saavedra, M. Perez-Victoria, Phys. Rev. D 84, 115013 (2011). arXiv:1105.4606 [hep-ph]

57. J.A. Aguilar-Saavedra, M. Perez-Victoria, JHEP 1109, 097 (2011). arXiv:1107.0841 [hep-ph]

58. E. Alvarez, L. Da Rold, J.I.S. Vietto, A. Szynkman, JHEP 1109, 007 (2011). arXiv:1107.1473 [hep-ph]
59. J.A. Aguilar-Saavedra, J. Santiago, Phys. Rev. D 85, 034021 (2012). arXiv:1112.3778 [hep-ph]

60. J.A. Aguilar-Saavedra, A. Juste, Phys. Rev. Lett. 109, 211804 (2012). arXiv:1205.1898 [hep-ph]

61. J.A. Aguilar-Saavedra, W. Bernreuther, Z.G. Si, Phys. Rev. D 86, 115020 (2012). arXiv:1209.6352 [hep-ph]

62. J.A. Aguilar-Saavedra, M. Perez-Victoria, J. Phys. Conf. Ser. 447, 012015 (2013). arXiv:1302.6618 [hep-ph]

63. M. Baumgart, B. Tweedie, JHEP 1308, 072 (2013). arXiv: 1303.1200 [hep-ph]

64. J.A. Aguilar-Saavedra, Phys. Lett. B 736, 132 (2014). arXiv:1405.1412 [hep-ph]

65. J. Drobnak, J.F. Kamenik, J. Zupan, Phys. Rev. D 86, 054022 (2012). arXiv:1205.4721 [hep-ph]

66. E. Coluccio Leskow, E. Alvarez, PoS ICHEP 2012, 201 (2013)

67. E. Alvarez, E.C. Leskow, Phys. Rev. D 86, 114034 (2012). arXiv:1209.4354 [hep-ph]

68. J. Drobnak, A.L. Kagan, J.F. Kamenik, G. Perez, J. Zupan, Phys. Rev. D 86, 094040 (2012). arXiv: 1209.4872 [hep-ph]

69. J.A. Aguilar-Saavedra, A. Juste, F. Rubbo, Phys. Lett. B 707, 92 (2012). arXiv:1109.3710 [hep-ph]

70. J.A. Aguilar-Saavedra, E. Alvarez, A. Juste, F. Rubbo, JHEP 1404, 188 (2014). arXiv:1402.3598 [hep-ph]

71. S. Kraml, H. Eberl, A. Bartl, W. Majerotto, W. Porod, Phys. Lett. B 386, 175 (1996). hep-ph/9605412

72. A. Djouadi, W. Hollik, C. Junger, Phys. Rev. D 55, 6975 (1997). hep-ph/9609419

73. J. Guasch. hep-ph/9906517

74. J. Guasch, W. Hollik, J. Sola, JHEP 0210, 040 (2002). hep-ph/0207364

75. J. Guasch. http://www.ffn.ub.es/guasch/progs/sfdecay/

76. L.J. Hall, R. Rattazzi, U. Sarid, Phys. Rev. D 50, 7048 (1994). hep-ph/9306309

77. M.S. Carena, M. Olechowski, S. Pokorski, C.E.M. Wagner, Nucl. Phys. B 426, 269 (1994). hep-ph/9402253

78. M.S. Carena, D. Garcia, U. Nierste, C.E.M. Wagner, Nucl. Phys. B 577, 88 (2000). hep-ph/9912516

79. J. Guasch, W. Hollik, S. Penaranda, Phys. Lett. B 515, 367 (2001). hep-ph/0106027

80. J. Guasch, P. Hafliger, M. Spira, Phys. Rev. D 68, 115001 (2003). hep-ph/0305101

81. K.-i. Hikasa, Y. Nakamura, Z. Phys. C 70, 139 (1996). [Erratumibid. C 71, 356 (1996)]. hep-ph/9501382

82. R.M. Godbole, L. Hartgring, I. Niessen, C.D. White, JHEP 1201, 011 (2012). arXiv:1111.0759 [hep-ph]

83. R.M. Godbole, K. Rao, S.D. Rindani, R.K. Singh, JHEP 1011, 144 (2010). arXiv:1010.1458 [hep-ph]

84. A. Prasath, R.M. Godbole, S.D. Rindani. arXiv:1405.1264 [hep$\mathrm{ph}]$

85. J. Shelton, Phys. Rev. D 79, 014032 (2009). arXiv:0811.0569 [hep-ph]

86. J.A. Aguilar-Saavedra, M. Perez-Victoria, JHEP 1105, 034 (2011). arXiv:1103.2765 [hep-ph]

87. A. Djouadi, G. Moreau, F. Richard, Phys. Lett. B 701, 458 (2011). arXiv:1105.3158 [hep-ph]

88. P. Ferrario, G. Rodrigo, Phys. Rev. D 78, 094018 (2008). arXiv:0809.3354 [hep-ph]

89. S. Jung, H. Murayama, A. Pierce, J.D. Wells, Phys. Rev. D 81 , 015004 (2010). arXiv:0907.4112 [hep-ph]

90. J. Shu, T.M.P. Tait, K. Wang, Phys. Rev. D 81, 034012 (2010). arXiv:0911.3237 [hep-ph]

91. A. Falkowski, G. Perez, M. Schmaltz, Phys. Rev. D 87, 034041 (2013). arXiv:1110.3796 [hep-ph]

92. A. Falkowski, M.L. Mangano, A. Martin, G. Perez, J. Winter, Phys. Rev. D 87, 034039 (2013). arXiv:1212.4003 [hep-ph] 
93. B. Gripaios, A. Papaefstathiou, B. Webber, JHEP 1311, 105 (2013). arXiv:1309.0810 [hep-ph]

94. P.Z. Skands et al., JHEP 0407, 036 (2004). hep-ph/0311123

95. E. Schlieckau, Plenary talk at the 7th International Workshop on Top Quark Physics (TOP 2014). https://indico.in2p3.fr/event/ 10114/session/11/contribution/17/material/slides/0

96. M. Carena, S. Heinemeyer, O. Stl, C.E.M. Wagner, G. Weiglein, Eur. Phys. J. C 73, 2552 (2013). arXiv:1302.7033 [hep-ph]

97. M.W. Cahill-Rowley, J.L. Hewett, A. Ismail, M.E. Peskin, T.G. Rizzo. arXiv:1305.2419 [hep-ph]

98. S. Chatrchyan et al., CMS Collaboration. Phys. Lett. B 725, 243 (2013). arXiv:1305.2390 [hep-ex]

99. S. Chatrchyan et al., CMS Collaboration. Eur. Phys. J. C 73, 2677 (2013). arXiv:1308.1586 [hep-ex]

100. G. Aad et al., ATLAS Collaboration. JHEP 1310, 189 (2013). arXiv:1308.2631 [hep-ex]

101. S. Chatrchyan et al., CMS Collaboration. Phys. Rev. Lett. 112, 161802 (2014). arXiv: 1312.3310 [hep-ex]

102. G. Aad et al., ATLAS Collaboration. JHEP 1406, 124 (2014). arXiv: 1403.4853 [hep-ex]

103. G. Aad et al. [ATLAS Collaboration], Eur. Phys. J. C 74 (2014) 6, 2883 arXiv:1403.5222 [hep-ex]

104. G. Aad et al., ATLAS Collaboration. JHEP 1406, 035 (2014). arXiv: 1404.2500 [hep-ex]

105. V. Khachatryan et al., CMS Collaboration. Phys. Lett. B 736, 371 (2014). arXiv:1405.3886 [hep-ex]

106. S. Heinemeyer, W. Hollik, G. Weiglein, Phys. Lett. B 455, 179 (1999). hep-ph/9903404

107. W. Barletta et al., arXiv:1310.0290 [physics.acc-ph]

108. K. Jakobs [ATLAS and CMS Collaborations], Nucl. Instrum. Meth. A 636, S1 (2011)

109. J. Germer, W. Hollik, J.M. Lindert, E. Mirabella, JHEP 1409, 022 (2014). arXiv:1404.5572 [hep-ph]

110. W. Hollik, E. Mirabella, JHEP 0812, 087 (2008). arXiv:0806.1433 [hep-ph]

111. W. Hollik, E. Mirabella, M.K. Trenkel, JHEP 0902, 002 (2009). arXiv:0810.1044 [hep-ph]

112. J. Germer, W. Hollik, E. Mirabella, M.K. Trenkel, JHEP 1008, 023 (2010). arXiv:1004.2621 [hep-ph]

113. J. Germer, W. Hollik, E. Mirabella, JHEP 1105, 068 (2011). arXiv:1103.1258 [hep-ph]
114. W. Beenakker et al., JHEP 1412, 023 (2014). arXiv:1404.3134 [hep-ph]

115. W. Beenakker et al., JHEP 0912, 041 (2009). arXiv:0909.4418 [hep-ph]

116. W. Beenakker et al., JHEP 1008, 098 (2010). arXiv:1006.4771 [hep-ph]

117. W. Beenakker et al., Int. J. Mod. Phys. A 26, 2637 (2011). arXiv:1105.1110 [hep-ph]

118. W. Beenakker et al., Phys. Rev. D 85, 075014 (2012). arXiv:1106.5647 [hep-ph]

119. W. Beenakker et al., JHEP 1201, 076 (2012). arXiv:1110.2446 [hep-ph]

120. W. Beenakker et al., JHEP 1310, 120 (2013). arXiv:1304.6354 [hep-ph]

121. M. Cacciari, M. Czakon, M. Mangano, A. Mitov, P. Nason, Phys. Lett. B 710, 612 (2012). arXiv:1111.5869 [hep-ph]

122. P. Brnreuther, M. Czakon, A. Mitov, Phys. Rev. Lett. 109, 132001 (2012). arXiv:1204.5201 [hep-ph]

123. M. Czakon, A. Mitov, JHEP 1212, 054 (2012). arXiv:1207.0236 [hep-ph]

124. M. Czakon, A. Mitov, JHEP 1301, 080 (2013). arXiv:1210.6832 [hep-ph]

125. M. Czakon, P. Fiedler, A. Mitov, Phys. Rev. Lett. 110(25), 252004 (2013). arXiv:1303.6254 [hep-ph]

126. N. Arkani-Hamed, P. Schuster, N. Toro, J. Thaler, L.T. Wang, B. Knuteson, S. Mrenna. hep-ph/0703088 [hep-ph]

127. J. Alwall, M.P. Le, M. Lisanti, J.G. Wacker, Phys. Rev. D 79, 015005 (2009). arXiv:0809.3264 [hep-ph]

128. J. Alwall, P. Schuster, N. Toro, Phys. Rev. D 79, 075020 (2009). arXiv:0810.3921 [hep-ph]

129. D.S.M. Alves, E. Izaguirre, J.G. Wacker, Phys. Lett. B 702, 64 (2011). arXiv:1008.0407 [hep-ph]

130. D.S.M. Alves, E. Izaguirre, J.G. Wacker, JHEP 1110, 012 (2011). arXiv:1102.5338 [hep-ph]

131. D. Alves et al., LHC New Physics Working Group Collaboration. J. Phys. G 39, 105005 (2012). arXiv:1105.2838 [hep-ph]

132. J. Alwall et al., JHEP 1407, 079 (2014). arXiv: 1405.0301 [hep-ph]

133. R.M. Godbole, S.D. Rindani, R.K. Singh, JHEP 0612, 021 (2006). hep-ph/0605100 